\title{
AN OVERVIEW OF KNOT FLOER HOMOLOGY
}

\author{
PETER OZSVÁTH AND ZOLTÁN SZABÓ
}

Knot Floer homology is an invariant for knots discovered by the authors 91 and, independently, Jacob Rasmussen [105]. The discovery of this invariant grew naturally out of studying how a certain three-manifold invariant, Heegaard Floer homology 92, changes as the three-manifold undergoes Dehn surgery along a knot. Since its original definition, thanks to the contributions of many researchers, knot Floer homology has emerged as a useful tool for studying knots in its own right. We give here a few selected highlights of this theory, and then move on to some new algebraic developments in the computation of knot Floer homology.

\section{Motivation For the CONStRuction}

Since the work of Donaldson, gauge theory has emerged as the central tool for understanding differential topology in dimension four. Donaldson's pioneering work from the 1980's used the moduli space of solutions to the anti-self-dual Yang-Mills equations - or "instantons" - to construct diffeomorphism invariants of four-dimensional manifolds [14, 16]. Donaldson used these invariants to discover completely unexpected phenomena in four-dimensional topology, including a deep connection between the smooth topology of algebraic surfaces and their algebraic geometry, leading to a number of breakthroughs in the field [15, 52, 34, 27, 40, 112, 20, 56.

A corresponding invariant for three-dimensional manifolds was introduced by Andreas Floer, instanton Floer homology, which is the homology group of a chain complex whose generators are $S U(2)$ representations of the fundamental group of the three-manifold $Y$ (modulo conjugation), and whose differential counts instantons on $\mathbb{R} \times Y$; see [29, 19]. Floer homology can be used as a tool for computing Donaldson's invariants [28].

Floer formulated his instanton homology theory as a kind of infinite-dimensional Morse theory, akin to his earlier Lagrangian Floer homology, which is an invariant for a symplectic manifold, equipped with a pair of Lagrangian submanifolds [30; see also [36]. In [1, Atiyah proposed a relationship between these two invariants, which is now known as the "Atiyah-Floer conjecture". The starting point of this conjecture is a three-manifold equipped with a Heegaard splitting. The "character variety" of the Heegaard surface $\Sigma$, which is the space of representations of $\pi_{1}(\Sigma)$ into $S U(2)$ modulo conjugation, is equipped with a pair of Lagrangian subspaces, the spaces of representations that extend over each handlebody. The Atiyah-Floer conjecture states that the Lagrangian Floer homology of these character varieties should agree with the instanton homology of the underlying three-manifold $Y$; compare [113]. This statement is still a little vague: $S U(2)$ instanton homology

PSO was supported by NSF grant number DMS-1405114.

ZSz was supported by NSF grant number DMS-1606571. 
is defined for three-manifolds with $H_{1}(Y ; \mathbb{Z})=0$, and the spaces involved on the symplectic side are singular. Nonetheless, the conjectured relationship has spurred a great deal of mathematical activity [21, 117].

In 1994, four-manifold topology was revolutionized by the introduction of the Seiberg-Witten equations, a new partial differential equation coming from physics [118]. The moduli spaces of solutions to these equations could be used to construct invariants of smooth four-manifolds, just as the anti-self-dual equations are used in Donaldson's theory. Many theorems proved earlier using Donaldson's invariants had easier proofs and generalizations using the newly introduced SeibergWitten invariants [17. The Seiberg-Witten invariants also elucidated the relationship between the differential topology of symplectic manifolds and their symplectic properties, resulting in Clifford Taubes' celebrated proof that identified the Gromov-Witten invariants of a symplectic manifold with their Seiberg-Witten invariants [114, 115, 116].

Considerable work went into formulating a three-dimensional analogue of the SeibergWitten invariants. A definitive construction was given by Peter Kronheimer and Tomasz Mrowka in their monograph [53]; see also [76, 35, 72].

Heegaard Floer homology [92 grew out of our attempts to concretely understand the geometric underpinnings of Seiberg-Witten theory. A motivating problem was to find the analogue of the "Atiyah-Floer conjecture": what Lagrangian Floer construction could possibly recapture the Seiberg-Witten invariants for three-manifolds? A clue was offered by the the following observation: the space of stationary solutions to the (suitably perturbed) Seiberg-Witten equations on $\mathbb{R} \times \Sigma$ is identified the moduli space of "vortices" on $\Sigma$ with some charge $d$, which in turn, by early work of Taubes, is identified with the $d$-fold symmetric product of $\Sigma$, the space of unordered $d$-tuples of points in $\Sigma$, denoted $\operatorname{Sym}^{d}(\Sigma)$.

It was proved in 92 that Heegaard Floer homology is a well-defined three-manifold invariant, enjoying many of the properties of Seiberg-Witten theory. Although it was designed to be isomorphic to construction in Seiberg-Witten theory, the conjectural equivalence of these two theories was verified many years after their formulation, in the work of Cagatay Kutluhan, Yi-Jen Lee, Taubes [60] and Vincent Colin, Paolo Ghiggini, and Ko Honda [10].

Acknowledgements. We would like to thank András Stipsicz for his suggestions on an early draft of this paper. The work of Simon Donaldson has had a great impact on our research. Both of our $\mathrm{PhD}$ theses were based on computing Donaldson's invariant for four-manifolds; and indeed his theory has served as an inspiration to us ever since!

\section{Statement of the symplectic constructions}

We sketch now the construction of Heegaard Floer homology, and its corresponding knot invariant. Before doing this, we recall some topological preliminaries.

Let $\Sigma$ be a surface of genus $g$. A complete set of attaching circles for $\Sigma$ is a $g$ tuple of pairwise disjoint, homologically linearly independent simple, closed curves. A complete set of attaching circles specifies a handlebody $U_{\gamma}$ whose boundary is 
identified with $\Sigma$, so that the attaching circles bound disjoint, embedded disks in $U_{\gamma}$.

A Heegaard splitting of a connected, closed, oriented three-manifold $Y$ is a decomposition of $Y$ as the union of two handlebodies, glued along their boundary. Combinatorially, a Heegaard splitting is specified by a Heegaard diagram, which consists of a triple $(\Sigma, \boldsymbol{\alpha}, \boldsymbol{\beta})$, where $\Sigma$ is an oriented surface, $\boldsymbol{\alpha}=\left\{\alpha_{1}, \ldots, \alpha_{g}\right\}$ and $\boldsymbol{\beta}=\left\{\beta_{1}, \ldots, \beta_{g}\right\}$ are two complete sets of attaching circles for $\Sigma$. Heegaard diagrams can be thought of from the perspective of Morse theory [77, 78], as follows. If $Y$ is equipped with a self-indexing Morse function $f$ and a gradient-like vector field $v$, we can let $\Sigma$ be $f^{-1}(3 / 2)$, and $\boldsymbol{\alpha}$ is the locus of points in $\Sigma$ that flow out of the index one critical points under $v$, and $\boldsymbol{\beta}$ is the locus of points in $\Sigma$ that flow into the the index two critical points. We will typically work with pointed Heegaard diagrams, which consist of data $\mathscr{H}=(\Sigma, \boldsymbol{\alpha}, \boldsymbol{\beta}, w)$, where $(\Sigma, \boldsymbol{\alpha}, \boldsymbol{\beta})$ is a Heegaard diagram, and $w \in \Sigma$ is an auxiliary basepoint in $\Sigma$ that is disjoint from all the $\alpha_{i}$ and the $\beta_{j}$. (See Figure 2 for a somewhat complicated Heegaard diagram for $S^{3}$, ignoring the extra basepoint labelled $z$.)

Inside $\operatorname{Sym}^{g}(\Sigma)$, there is a pair of $g$-dimensional tori

$$
\mathbb{T}_{\alpha}=\alpha_{1} \times \cdots \times \alpha_{g} \quad \text { and } \quad \mathbb{T}_{\beta}=\beta_{1} \times \cdots \times \beta_{g} ;
$$

e.g. $\mathbb{T}_{\alpha}$ is the space of $g$-tuples of points in $\Sigma$, so that each point lies on some $\alpha_{i}$ and no two points lie on the same $\alpha_{i}$. The basepoint gives rise to a real codimension two submanifold $V_{w} \subset \operatorname{Sym}^{g}(\Sigma)$, consisting of those $g$-tuples of points $\mathbf{x}$ that include the point $w$.

The intersection points $\mathbb{T}_{\alpha} \cap \mathbb{T}_{\beta}$ are called Heegaard states for the diagram $\mathscr{H}$, and they are denoted $\mathfrak{S}(\mathscr{H})$. Explicitly, if we think of the $\alpha$ - and $\beta$-circles as numbered by $\{1, \ldots, g\}$, then Heegaard states are partitioned according to permutations $\sigma$ on $\{1, \ldots, g\}$. The Heegaard states of type $\sigma$ correspond to points in the Cartesian product

$$
\left(\alpha_{1} \cap \beta_{\sigma(1)}\right) \times \cdots \times\left(\alpha_{g} \cap \beta_{\sigma(g)}\right) .
$$

A complex structure on $\Sigma$ naturally induces a complex structure on the $g$-fold symmetric product $\operatorname{Sym}^{g}(\Sigma)$. In fact, the $g$-fold symmetric product $\operatorname{Sym}^{g}(\Sigma)$ can be given a Kähler structure so that the tori $\mathbb{T}_{\alpha}$ and $\mathbb{T}_{\beta}$ are Lagrangian [101. Versions of the Heegaard Floer homology of $Y$ correspond to variants of Lagrangian Floer homology for $\mathbb{T}_{\alpha}$ and $\mathbb{T}_{\beta}$ in $\operatorname{Sym}^{g}(\Sigma)$, which depend on how one counts pseudo-holomorphic disks which interact with the subspace $V_{w}$.

Choosing an almost-complex structure compatible with the symplectic structure on $\operatorname{Sym}^{g}(\Sigma)$, one can consider pseudo-holomorphic disks, as introduced by Gromov [42. For fixed Heegaard states $\mathbf{x}$ and $\mathbf{y}$, the pseudo-holomorphic disks in $\operatorname{Sym}^{g}(\Sigma)$ connecting $\mathbf{x}$ to $\mathbf{y}$ can be organized into homotopy classes of maps from the unit disk $\mathbb{D}$ in the complex plane to $\operatorname{Sym}^{g}(\Sigma), u: \mathbb{D} \rightarrow \operatorname{Sym}^{g}(\Sigma)$, satisfying the following boundary conditions: $u$ maps $-i$ to $\mathbf{x}, i$ to $\mathbf{y}$, and $x+i y=z \in \partial \mathbb{D}$ with $x \geq 0$ to $\mathbb{T}_{\alpha}$ and $x \leq 0$ to $\mathbb{T}_{\beta}$. We denote the space of homotopy classes of such maps by $\pi_{2}(\mathbf{x}, \mathbf{y})$. Since $w$ is disjoint from the $\alpha_{i}$ and $\beta_{j}$, there is a well-defined map $n_{w}: \pi_{2}(\mathbf{x}, \mathbf{y}) \rightarrow \mathbb{Z}$ which is given as the algebraic intersection number of a generic $u$ representing $\phi \in \pi_{2}(\mathbf{x}, \mathbf{y})$ with the oriented submanifold $V_{w}$. The moduli space of pseudo-holomorphic disks representing the homotopy 
class $\phi \in \pi_{2}(\mathbf{x}, \mathbf{y})$ is denoted $\mathscr{M}(\phi)$. This admits a natural action by $\mathbb{R}$, thought of as the holomorphic automorphisms of $\mathbb{D}$ preserving $\pm i$.

The simplest version of Heegaard Floer homology is the homology of a chain complex $\widehat{\mathrm{CF}}(\mathscr{H})$, thought of a vector space over the field $\mathbb{F}$ with two elements. Generators of this chain complex are the Heegaard states, and its differential counts pseudo-holomorphic disks that are disjoint from $V_{w}$; more formally, $\widehat{\mathrm{CF}}(\mathscr{H})$ is the vector space generated $\mathfrak{S}(\mathscr{H})$, equipped with the differential

$$
\widehat{\partial}(\mathbf{x})=\sum_{\mathbf{y} \in \mathfrak{S}} \sum_{\left\{\phi \in \pi_{2}(\mathbf{x}, \mathbf{y}) \mid n_{w}(\phi)=0, \mu(\phi)=1\right\}} \#\left(\frac{\mathcal{M}(\phi)}{\mathbb{R}}\right) \cdot \mathbf{y} .
$$

Here, $\mu(\phi)$ is the Maslov index of the homotopy class $\phi$ [107, 36]; see [62, for a very useful formulation in terms of the Heegaard diagram. As is standard in Floer theory [33, 36, to make sense of the definition, the $\bar{\partial}$-equations need to be perturbed suitably to make the moduli spaces transverse. This chain complex has a refinement $\mathrm{CF}^{-}(\mathscr{H})$, which is a module over the polynomial algebra $\mathbb{F}[U]$, and whose differential is defined by

$$
\partial^{-}(\mathbf{x})=\sum_{\mathbf{y} \in \mathfrak{S}} \sum_{\left\{\phi \in \pi_{2}(\mathbf{x}, \mathbf{y}) \mid \mu(\phi)=1\right\}} \#\left(\frac{\mathscr{M}(\phi)}{\mathbb{R}}\right) \cdot U^{n_{w}(\phi)} \mathbf{y} .
$$

The $U=0$ specialization of this chain complex is $\widehat{\mathrm{CF}}(\mathscr{H})$. (Both complexes can in fact be defined over $\mathbb{Z}$ coefficients; see [92].)

The main theorem of 92 states that the homology of $\mathrm{CF}^{-}(\mathscr{H})$ (and $\widehat{\mathrm{CF}}(\mathscr{H})$ ) is an invariant of the underlying closed, oriented three-manifold $Y$ represented by $\mathscr{H}$.

Heegaard Floer homology has an extension to knots $K \subset Y$ in a three-manifold, called knot Floer homology, which was discovered independently by Jacob Rasmussen [105] and by us [85]. For this version, start with a doubly-pointed Heegaard diagram $\mathscr{H}=(\Sigma, \boldsymbol{\alpha}, \boldsymbol{\beta}, w, z)$, where here the two basepoints $w$ and $z$ in $\Sigma$ are both chosen to be disjoint from the $\alpha_{i}$ and the $\beta_{i}$. This data specifies an oriented knot inside the three-manifold $Y$ specified by the Heegaard diagram $(\Sigma, \boldsymbol{\alpha}, \boldsymbol{\beta})$. The knot is constructed by the following procedure. Connect $w$ to $z$ in $\Sigma$ by an arc $a$ that is disjoint from the $\alpha$-curves, and push the interior of resulting arc into the $\alpha$-handlebody; similarly, connect $w$ and $z$ by another arc in $\Sigma$ that is disjoint from the $\beta$-curves and push the interior of that into the $\beta$-handlebody to get $b$. The knot $K$ is obtained as $a \cup b$. It can be oriented by the convention that $\partial a=z-w=-\partial b$.

The simplest version of knot Floer homology is the homology of a chain complex $\widehat{\mathrm{CFK}}(\mathscr{H})$, once again generated by Heegaard states (in a doubly-pointed Heegaard diagram $\mathscr{H}$ representing $K$ ), with differential given by

$$
\widehat{\partial}_{K}(\mathbf{x})=\sum_{\mathbf{y} \in \mathfrak{S}} \sum_{\left\{\phi \in \pi_{2}(\mathbf{x}, \mathbf{y}) \mid n_{w}(\phi)=0=n_{z}(\phi), \mu(\phi)=1\right\}} \#\left(\frac{M(\phi)}{\mathbb{R}}\right) \mathbf{y} .
$$

For simplicity, we hereafter restrict attention to the case where the ambient threemanifold is $S^{3}$. Dropping the requirement that $n_{z}(\phi)=0$ gives the chain complex representing $\widehat{\mathrm{HF}}\left(S^{3}\right)$, which is a one-dimensional vector space. Knot Floer complex is equipped with two gradings, the Maslov grading and the Alexander grading, 
induced by functions

$$
M: \mathfrak{S}(\mathscr{H}) \rightarrow \mathbb{Z} \quad \text { and } \quad A: \mathfrak{S}(\mathscr{H}) \rightarrow \mathbb{Z}
$$

that are characterized as follows.

The function $M$ satisfies the property that if $\mathbf{x}$ and $\mathbf{y}$ are any two Heegaard states, and $\phi \in \pi_{2}(\mathbf{x}, \mathbf{y})$ is a homotopy class of Whitney disks, then

$$
M(\mathbf{x})-M(\mathbf{y})=\mu(\phi)-2 n_{w}(\phi) .
$$

This specifies $M$ uniquely up to an overall additive constant. The function $M$ induces a $\mathbb{Z}$-valued grading on $\widehat{\mathrm{CF}}\left(S^{3}\right)$ for which the differential $\widehat{\partial}$ drops grading by one; thus there is an induced grading on $\widehat{\mathrm{HF}}\left(S^{3}\right) \cong \mathbb{F}$. The additive indeterminacy on $M$ is pinned down by requiring that $\widehat{\mathrm{HF}}\left(S^{3}\right)$ is supported in Maslov grading equal to zero.

The function $A$ satisfies the property that if $\mathbf{x}$ and $\mathbf{y}$ are any two Heegaard states, and $\phi \in \pi_{2}(\mathbf{x}, \mathbf{y})$ is a homotopy class of Whitney disks, then

$$
A(\mathbf{x})-A(\mathbf{y})=n_{z}(\phi)-n_{w}(\phi) .
$$

Once again, this specifies $A$ up to an overall additive constant; and the differential $\widehat{\partial}_{K}$ preserves the corresponding splitting of $\widehat{\mathrm{CFK}}(\mathscr{H})$ specified by the Alexander grading.

The Maslov and Alexander functions induce a bigrading on $\widehat{\mathrm{CFK}}(\mathscr{H})$,

$$
\widehat{\mathrm{CFK}}(\mathscr{H})=\bigoplus_{d, s \in \mathbb{Z}} \widehat{\mathrm{CFK}}_{d}(\mathscr{H}, s)
$$

where $\widehat{\mathrm{CFK}}_{d}(\mathscr{H}, s)$ is generated by those states $\mathbf{x}$ with $M(\mathbf{x})=d$ and $A(\mathbf{x})=s$. The differential satisfies

$$
\widehat{\partial}_{K}: \widehat{\mathrm{CFK}}_{d}(\mathscr{H}, s) \rightarrow \widehat{\mathrm{CFK}}_{d-1}(\mathscr{H}, s),
$$

and therefore the bigrading descends to homology

$$
\widehat{\operatorname{HFK}}(\mathscr{H})=\bigoplus_{d, s \in \mathbb{Z}} \widehat{\operatorname{HFK}}_{d}(\mathscr{H}, s)
$$

The bigraded chain complex $\widehat{\mathrm{CFK}}(\mathscr{H})$ has a graded Euler characteristic, which is a Laurent polynomial with integral coefficients, in a formal variable $t$, defined by

$$
\chi(\widehat{\mathrm{CFK}}(K))=\sum_{d, s}(-1)^{d} \operatorname{dim} \widehat{\mathrm{CFK}}_{d}(K, s) t^{s}=\sum_{d, s}(-1)^{d} \operatorname{dim} \widehat{\mathrm{HFK}}_{d}(K, s) t^{s} .
$$

This graded Euler characteristic coincides with the Alexander polynomial $\Delta_{K}(t)$ :

$$
\chi(\widehat{\mathrm{CFK}}(K)) \doteq \Delta_{K}(t),
$$

where here $\doteq$ means that the two polynomials agree up to overall factors of $t$ [91]. The additive indeterminacy in $A$ is eliminated by requiring that the graded Euler characteristic is symmetric in $t$; i.e. Equation (2.1) holds with equality in place of $\doteq$. 

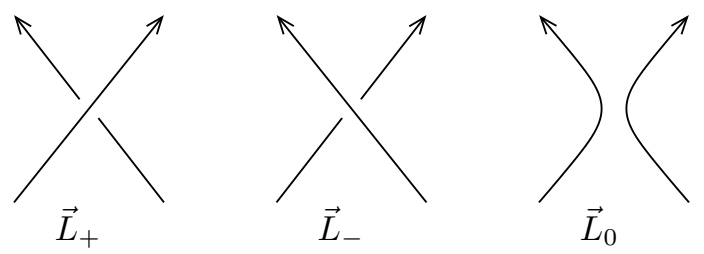

Figure 1. Crossing conventions in the skein relation.

The information in the bigraded vector space $\widehat{\mathrm{HFK}}(K)$ is encoded in its Poincaré polynomial, a polynomial with non-negative integral coefficients in two formal variables $q$ and $t$, defined by

$$
P_{K}(q, t)=\sum_{d, s} \operatorname{dim} \widehat{\operatorname{HFK}}_{d}(K, s) q^{d} t^{s} .
$$

Specializing $P_{K}$ to $q=-1$ gives the graded Euler characteristic; i.e. $P_{K}(-1, t)=$ $\Delta_{K}(t)$.

The construction described above is analytic in nature: the generators are combinatorial, but differentials count pseudo-holomorphic disks. Knot Floer homology has a number of different, more computationally approachable formulations. We will return to this point, but first, we outline some properties and applications of the invariant.

\section{First PROPERTIES}

We describe now some basic properties of knot Floer homology, contrasting them with corresponding properties for the Alexander polynomial.

Suppose that $K_{+}$and $K_{-}$are two knots with a projection that differs in exactly one crossing, as shown in the first two pictures of Figure 1. Then, we can resolve the crossing to obtain a new oriented link with two components, the third picture in that figure. More generally, if $\vec{L}_{+}, \vec{L}_{-}$, and $\vec{L}_{0}$ are three oriented links that differ as in that figure, we say that they form a skein triple. The Alexander polynomial for knots can be extended to oriented links, and that extension satisfies the following skein relation for any skein triple $\vec{L}_{+}, \vec{L}_{-}, \vec{L}_{0}$ :

$$
\Delta_{\vec{L}_{+}}(t)-\Delta_{\vec{L}_{-}}(t)=\left(t^{1 / 2}-t^{-1 / 2}\right) \Delta_{\vec{L}_{0}}(t) .
$$

This relation gives a helpful inductive computational procedure for the Alexander polynomial. In fact, it was observed by John Conway [11 that the Alexander polynomial (for oriented links) is uniquely characterized by the above skein relation, and the normalization for the unknot $\mathcal{U}$, which states that $\Delta_{\mathscr{U}}(t)$ is the constant polynomial 1. Extending knot Floer homology to links, the skein relation has the following analogue:

Theorem 3.1. If $\vec{L}_{+}, \vec{L}_{-}$, and $\vec{L}_{0}$ are three oriented links that fit into a skein triple, there is a corresponding exact triangle relating their bigraded knot Floer homologies. When the two strands meeting at $\vec{L}_{+}$belong to the same component of 
$\vec{L}_{+}$, the triangle has the form

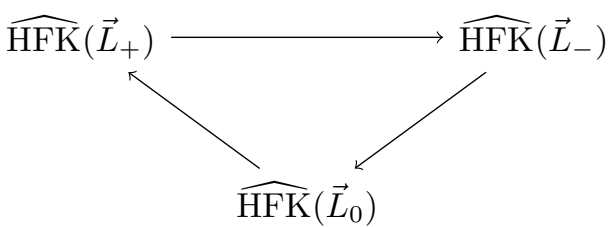

When the strands in $\vec{L}_{+}$belong to different components, there is a similar triangle, except that $\widehat{\mathrm{HFK}}\left(\vec{L}_{0}\right)$ is tensored with an appropriately graded four-dimensional bigraded vector space.

See [91] and [84, Chapter 9] for a precise statement (with specified bigradings). The above result should be compared with Floer's exact triangle for instanton homology; compare [31, 32, 6].

The Alexander polynomial polynomial is multiplicative under connected sum. This has the following generalization to the case of knot Floer homology:

Proposition 3.2. If $K_{1}$ and $K_{2}$ are two knots, then $\widehat{\mathrm{HFK}}\left(K_{1} \# K_{2}\right)$ is obtained as the graded tensor product of the bigraded vector spaces $\widehat{\operatorname{HFK}}\left(K_{1}\right)$ and $\widehat{\operatorname{HFK}}\left(K_{2}\right)$; i.e. $P_{K_{1} \# K_{2}}(q, t)=P_{K_{1}}(q, t) \cdot P_{K_{2}}(q, t)$

Recall that a knot is called alternating if it has a diagram with the property that crossings alternate between over- and under-crossings as one follows the projection. By a classical theorem of Cromwell and Murasugi [12, 80, the Alexander polynomial of an alternating knot is special: its coefficients alternate in sign. This has the following analogue for knot Floer homology [88]; see also [104, 73].

Theorem 3.3. If $K$ is an alternating knot, then the knot Floer homology for $K$ is determined by its Alexander polynomial $\Delta_{K}(t)$ and its signature $\sigma(K)$, by the formula

$$
P_{K}(q, t)=q^{\frac{\sigma}{2}} \cdot \Delta_{K}(q t) .
$$

Knot Floer homology can be given more algebraic structure. For example, there is a version which is a free chain complex over $\mathbb{F}[U], \mathrm{CFK}^{-}(\mathscr{H})$, with differential

$$
\partial_{K}^{-}(\mathbf{x})=\sum_{\mathbf{y} \in \mathfrak{S}} \sum_{\left\{\phi \in \pi_{2}(\mathbf{x}, \mathbf{y}) \mid n_{z}(\phi)=0, \mu(\phi)=1\right\}} \#\left(\frac{\mathscr{M}(\phi)}{\mathbb{R}}\right) U^{n_{w}(\phi)} \mathbf{y} .
$$

Extending the Maslov and Alexander grading so that multiplication by $U$ drops Maslov grading by 2 and Alexander grading by 1, we have that

$$
\partial_{K}^{-}: \operatorname{CFK}_{d}^{-}(\mathscr{H}, s) \rightarrow \mathrm{CFK}_{d-1}^{-}(\mathscr{H}, s) \quad U: \mathrm{CFK}_{d}^{-}(\mathscr{H}, s) \rightarrow \mathrm{CFK}_{d-2}^{-}(\mathscr{H}, s-1) .
$$

Thus, the homology $\operatorname{HFK}^{-}(\mathscr{H})$ inherits the structure of a bigraded $\mathbb{F}[U]$-module.

Proposition 3.4. The bigraded module $\operatorname{HFK}^{-}(K)$ is finitely generated; in fact, it consists of direct summands of the form $\mathbb{F}[U] / U^{m}$ for various choices of $m$, and a single free summand $\mathbb{F}[U]$.

The above proposition is clear from the original definitions of knot Floer homology [91, 105]; see [84, Chapter 7] for a more precise reference. 
Proposition 3.4 shows that $\mathrm{HFK}^{-}(K)$ always contains $U$-non-torsion elements, i.e. elements $\xi \in \operatorname{HFK}^{-}(K)$ with $U^{m} \cdot \xi \neq 0$ for all $m$. Thus, there is a natural numerical invariant of knots $K$, denoted $\tau(K)$ which is defined as -1 times the maximal Alexander grading of any non-torsion class $\xi \in \operatorname{HFK}^{-}(K)$.

\section{TOPOLOGicAl APPLiCATIONS}

Knot Floer homology was originally envisioned as a tool for computing the Heegaard Floer homology groups of three-manifolds obtained as surgeries on a given knot. If

$K$ is a knot in $S^{3}$, a "surgery formula" expresses the Heegaard Floer homology $\widehat{\mathrm{HF}}$ for three-manifolds obtained as surgeries on $K$ in terms of another variant of knot Floer homology $\operatorname{HFK}(K)$ defined over the ring $\mathscr{R}=\mathbb{F}[U, V] / U V=0$. This knot invariant is the homology of a chain complex, $\operatorname{CFK}(\mathscr{H})$, which is freely generated (over $\mathscr{R}$ ) by Heegaard states, and whose differential is given by

$$
\partial(\mathbf{x})=\sum_{\mathbf{y} \in \mathfrak{S}} \sum_{\left\{\phi \in \pi_{2}(\mathbf{x}, \mathbf{y}) \mid \mu(\phi)=1\right\}} \#\left(\frac{\mathscr{M}(\phi)}{\mathbb{R}}\right) \cdot U^{n_{w}(\phi)} V^{n_{z}(\phi)} \mathbf{y} .
$$

As in the case of $\mathrm{HFK}^{-}$, the homology module inherits a bigrading: in the present case, $U$ drops Alexander grading by one, and $V$ raises it by one.

We do not state the surgery formula here (see [96, 97]), but we do give a consequence:

Theorem 4.1. 94] Suppose that $K$ is a knot with the property that for some rational number $r \in \mathbb{Q}$, the three-manifold $S_{r}^{3}(K)$ is a lens space. Then, all the coefficients of the Alexander polynomial are \pm 1 or 0 ; in fact, the non-zero ones alternate in sign. Thus, we can write

$$
\Delta_{K}(t)=\sum_{k=0}^{n}(-1)^{k} t^{\alpha_{k}}
$$

where $\left\{\alpha_{k}\right\}_{k=0}^{n}$ is a decreasing sequence of integers. Moreover, when $r>0$, the knot Floer homology of $K$ is determined by this Alexander polynomial, as follows. There is a sequence of integers $\left\{m_{k}\right\}_{k=0}^{n}$ determined by the formulae:

$$
\begin{aligned}
m_{0} & =0 \\
m_{2 k} & =m_{2 k-1}-1 \\
m_{2 k+1} & =m_{2 k}-2\left(\alpha_{2 k}-\alpha_{2 k+1}\right)+1,
\end{aligned}
$$

so that

$$
P_{T_{p, q}}(q, t)=\sum_{k=0}^{n} q^{m_{k}} t^{\alpha_{k}}
$$

In particular, for each $s \in \mathbb{Z}, \widehat{\mathrm{HFK}}_{*}(K, s)$ has dimension 0 or 1 .

Recall that any knot $K \subset S^{3}$ can be realized as the boundary of an orientable surface $F$ embedded in $S^{3}$. Such a surface is called a Seifert surface for $K$, and the minimal genus of any Seifert surface for $K$ is called the Seifert genus of $K$. It is a classical result that the degree of the Alexander polynomial gives a lower bound for the Seifert genus of a knot. This result has a sharpening for knot Floer homology, which is inspired by work of Kronheimer and Mrowka [57]: 
Theorem 4.2. 90] Knot Floer homology detects the Seifert genus $g(K)$ of a knot $K$, in the sense that

$$
g(K)=\min \left\{s \mid \widehat{\operatorname{HFK}}_{*}(K, s) \neq 0\right\} .
$$

Our original proof of the above theorem relied on many results in low-dimensional topology. To start with, Gabai's theory of sutured manifolds equips the zero-surgery with a taut foliation [37. A theorem of Eliashberg and Thurston 24 provides the product $[-1,1] \times S_{0}^{3}(K)$ with a symplectic structure, which is suitably convex at the boundary. A theorem of Eliashberg [23] and Etnyre 25] embeds this symplectic cylinder in a closed symplectic manifold $X$. Donaldson's Lefschetz pencils [18] on symplectic manifolds then provides a suitable two-handle decomposition on $X$ for which we can prove that the Heegaard Floer four-manifold invariant is nonzero 93. A surgery formula relating knot Floer homology with the Heegaard Floer homology of the 0 -surgery then gives the required non-vanishing theorem for knot Floer homology.

Juhász has an elegant proof of the above result [45] that bypasses most of the above machinery (still building on Gabai's sutured hierarchy), using his sutured Floer homology [44].

Theorem 4.2 has the following corollary:

Corollary 4.3. [90] Knot Floer homology detects the unknot, in the sense that $\widehat{\mathrm{HFK}}(K)$ has dimension one if and only if $K$ is the unknot.

The above corollary underscores how far knot Floer homology goes beyond the Alexander polynomial: there are infinitely many knots with trivial Alexander polynomial.

Theorem 4.1 has a more precise statement, which expresses the sequence $\left\{\alpha_{k}\right\}$ concretely in terms of the surgery coefficient $r$ and the resulting lens space $L(p, q)$ [94]. In [90, we combine this result with Corollary 4.3, to obtain the following result, first proved using Seiberg-Witten theory in our joint work with Kronheimer and Mrowka:

Corollary 4.4. [59] If $K \subset S^{3}$ is a knot with the property that $S_{r}^{3}(K) \cong \mathbb{R}^{3}$, then $K$ is the unknot.

See 41 for a vast generalization.

A final property of knot Floer homology motivated by the Alexander polynomial is based on the classical result that the Alexander polynomial of a fibered knot is monic. This has an analogue for knot Floer homology: if $K$ is a fibered knot with Seifert genus $g=g(K)$, then

$$
\widehat{\operatorname{HFK}}_{*}(K, g)=\bigoplus_{d \in \mathbb{Z}} \widehat{\operatorname{HFK}}_{d}(K, g)
$$

is one-dimensional [93]. This fact has the following remarkable converse, due to Paolo Ghiggini when $g=1$ and $\mathrm{Yi}$ Ni when $g>1$ :

Theorem 4.5. 38, 82, If $\widehat{\mathrm{HFK}}_{*}(K, g)$ is one-dimensional, then $K$ is fibered.

See also [45]. 
Ni's theorem, combined with Theorem 4.1, immediately gives the following:

Corollary 4.6. [82] If $K \subset S^{3}$ is a knot with the property that $S_{r}^{3}(K)$ is a lens space, then $K$ is fibered.

So far, we have focused on applications on the simplest variant of knot Floer homology, $\widehat{\operatorname{HFK}}(K)$. The version $\operatorname{HFK}^{-}(K)$, with its module structure over $\mathbb{F}[U]$, has further applications to the unknotting number and the slice genus of a knot, which we recall here. Thinking of $S^{3}$ as a boundary of the four-ball $B^{4}$, one can consider slice surfaces: smoothly embedded surfaces in $B^{4}$, so that $F \backslash \partial F$ is mapped to $B^{4} \backslash \partial B^{4}=S^{3}$, and $\partial F$ is mapped to $K \subset S^{3}$. The slice genus of a knot $K$, denoted $g_{4}(K)$, is the minimal genus of any slice surface for $K$. A knot is called a slice knot if its slice genus is 0. Clearly, the Seifert genus of $K$ bounds the slice genus of $K: g_{4}(K) \leq g(K)$.

An unknotting of $K$ is a sequence of knots $K=K_{0}, K_{1}, \ldots, K_{n}$, where $K_{i}$ is obtained from $K_{i-1}$ by changing one crossing, so that $K_{n}$ is the unknot. The unknotting number of $K$, denoted $u(K)$, is the minimal length of any unknotting for $K$. An $n$-step unknotting for $K$ naturally gives rise to an immersed surface in $B^{4}$ with $n$ double points. Resolving these double-points, we can find a slice surface for $K$ with genus $n$. This proves the bound $g_{4}(K) \leq u(K)$.

The module structure $\operatorname{HFK}^{-}(K)$, and specifically the associated integral invariant $\tau$, gives a lower bound on the slice genus according to the following:

Theorem 4.7. For any knot $K \subset S^{3},|\tau(K)| \leq g_{4}(K)$.

The above is proved in [89]; see also [105] for other similar bounds. Sucharit Sarkar gave a combinatorial proof of Theorem 4.7 from the perspective of "grid diagrams"; see [108]; see also [84, Chapter 8].

Direct computation shows that for the $(p, q)$ torus knot $T_{p, q}, \tau\left(T_{p, q}\right)=\frac{(p-1)(q-1)}{2}$. Thus, Theorem 4.7 gives another verification of following theorem of Kronheimer and Mrowka, first conjectured by Milnor [79]:

Theorem 4.8. [55] For relatively prime integers $p$ and $q$, the torus knot $T_{p, q}$ has

$$
u\left(T_{p, q}\right)=g_{4}\left(T_{p, q}\right)=\frac{(p-1)(q-1)}{2} .
$$

It is easy to see that the quantity appearing in the above theorem also coincides with the Seifert genus of $T_{p, q}$. Kronheimer and Mrowka's proof of the above theorem used Donaldson invariants. A number of alternative proofs have emerged since. Rasmussen [106] gave the first combinatorial proof, using the algebraic structure on Khovanov's knot invariants; see also see 108.

There are non-orientable analogues of the slice genus, defined as follows. Consider possibly non-orientable surfaces $F$ embedded in $B^{4}$, meeting $S^{3}$ along $K$, and let $\gamma_{4}(K)$, the non-orientable 4 -genus of $K$ denote the minimal complexity, as measured by the dimension of $H_{1}(F ; \mathbb{F})$, for all such choices of $F$. For example, the torus knot $T_{2,2 n+1}$ bounds a $n+\frac{1}{2}$-twisted Möbius strip, so $\gamma_{4}\left(T_{2,2 n+1}\right)=1$.

Prior to 2012, the best lower bound on $\gamma_{4}$ for any knot was 3 . The situation was vastly improved by the following theorem of Joshua Batson: 
Theorem 4.9. 4] The non-orientable 4-genus can be arbitrarily large; for example, $\gamma_{4}\left(T_{2 k, 2 k-1}\right)=k-1$.

Batson's proof goes by constructing an explicit surface with stated complexity, to give an upper bound on $\gamma_{4}\left(T_{2 k, 2 k-1}\right)$. Next, he gives a lower bound on $\gamma_{4}\left(T_{2 k, 2 k-1}\right)$ via a Heegaard Floer invariant associated to surgeries on the knot.

An alternative proof of the above theorem is given in joint work of András Stipsicz and the authors 83, using another variant of knot Floer homology. This version is the homology of a chain complex $\operatorname{CFK}^{\prime}(\mathscr{H})$ which, like $\mathrm{CFK}^{-}(\mathscr{H})$, is freely generated over $\mathbb{F}[U]$ by the Heegaard states; but it is equipped with a differential

$$
\partial_{K}^{\prime} \mathbf{x}=\sum_{\mathbf{y} \in \mathfrak{S}_{\left\{\phi \in \pi_{2}(\mathbf{x}, \mathbf{y}) \mid \mu(\phi)=1\right\}}} \#\left(\frac{\mathscr{M}(\phi)}{\mathbb{R}}\right) U^{n_{w}(\phi)+n_{z}(\phi)} \mathbf{y} .
$$

This complex is equipped with the single grading $\delta(\mathbf{x})=M(\mathbf{x})-A(\mathbf{x})$. It is straightforward to check that $\partial_{K}^{\prime}$ drops $\delta$-grading by 1 , as does multiplication by $U$. Proposition 3.4 has the following analogue:

Proposition 4.10. [83, Proposition 3.5] The bigraded module $\operatorname{HFK}^{\prime}(K)$ is finitely generated; in fact, it consists of direct summands of the form $\mathbb{F}[U] / U^{m}$ for various choices of $m$, and a single free summand $\mathbb{F}[U]$.

We can now define $v(K)$ to be the maximal $\delta$-grading of any $U$-non-torsion element in $\operatorname{HFK}^{\prime}(K)$. Theorem 4.9 can be proved via a computation of $v\left(T_{2 k, 2 k-1}\right)$, combined with the following bound on the non-orientable 4 -genus in terms of $v$, analogous to Theorem 4.7 .

Theorem 4.11. 83] For any knot $K \subset S^{3},\left|v(K)-\frac{\sigma(K)}{2}\right| \leq \gamma_{4}(K)$.

Analyzing the slice genus is a place where smooth four-dimensional topology has a clear interaction with knot theory. The slice surfaces whose genus is minimized in the definition are thought of as smoothly embedded in $B^{4}$. Relaxing this requirement, we could ask for locally flat, topologically embedded surfaces, to obtain an analogous numerical knot invariant, called the topological slice genus, $g_{4}^{\text {top }}(K)$.

In a related direction, one can say that two knots $K_{1}$ and $K_{2}$ are concordant if there is an embedded annulus $F$ in $[1,2] \times S^{3}$ so that $F \cap\left(\{i\} \times S^{3}\right)$ is the knot $K_{i}$; or, equivalently, if $K_{1} \# \mathfrak{m}\left(K_{2}\right)$ is a slice knot, where here $\mathfrak{m}(K)$ denotes the mirror of $K$. The connected sum operation endows this set with the structure of an Abelian group, called the smooth concordance group $\mathscr{C}$. If we require the annulus to be only topologically embedded, or equivalently, if we require $K_{1} \# \mathfrak{m}\left(K_{2}\right)$ to be only topologically slice, we obtain another group, the topological concordance group, denoted $\mathscr{C}^{\text {top }}$. There is a canonical homomorphism $\mathscr{C} \rightarrow \mathscr{C}^{\text {top }}$, whose kernel is the subgroup of topologically slice knots, $\mathscr{C}_{T S}$.

Litherland [68] showed that $\mathscr{C}^{\text {top }}$ contains a direct summand of $\mathbb{Z}^{\infty}$. According to a theorem of Freedman, any knot with $\Delta_{K}(t)=1$ is topologically slice. Using Donaldson's diagonalizability theorem, Andrew Casson showed that $\mathscr{C}_{T S}$ is nontrivial; see [9]. In fact, the 0-twisted Whitehead double of the trefoil, a knot for which $\tau(K)=1$, gives a $\mathbb{Z}$-direct summand in $\mathscr{C}_{T S}$ [69. 
Using gauge theory, in 1995 Endo exhibited a $\mathbb{Z}^{\infty}$ subgroup of $\mathscr{C}_{T S}$. In 2012 , Jen Hom [43] went further, exhibiting a $\mathbb{Z}^{\infty}$ direct summand in $\mathscr{C}_{T S}$ by constructing infinitely many linearly independent concordance homomorphisms from $\mathscr{C}_{T S}$ to $\mathbb{Z}$. Her construction uses an invariant $\epsilon$, which can be viewed as derived from knot Floer homology $\operatorname{HFK}(K)$ over $\mathbb{F}[U, V] / U V$. Using $\epsilon$, she introduces an equivalence relation on the knot Floer complexes, to form a totally ordered Abelian group. The homomorphism are then provided by the axiom of choice.

In joint work with Stipsicz [99], we constructed another collection of homomorphisms to $\mathbb{Z}$, using a one-parameter deformation of knot Floer homology $\operatorname{tHFK}(K)$. Specifically, for each rational $t=\frac{p}{q} \in[0,2]$, there is a chain complex $\operatorname{tCFK}(K)$, freely generated over $\mathbb{F}\left[v^{1 / q}\right]$ by Heegaard states, whose differential now has the form

$$
\partial_{K}^{t} \mathbf{x}=\sum_{\mathbf{y} \in \mathfrak{S}} \sum_{\left\{\phi \in \pi_{2}(\mathbf{x}, \mathbf{y}) \mid \mu(\phi)=1\right\}} \#\left(\frac{\mathscr{M}(\phi)}{\mathbb{R}}\right) v^{t n_{w}(\phi)+(2-t) n_{z}(\phi)} \mathbf{y} .
$$

This complex is graded by $\operatorname{gr}_{t}(\mathbf{x})=M(\mathbf{x})-t A(\mathbf{x})$, so that multiplication by $v$ drops grading by 1 . When $t=0$, the complex is independent of the knot, and its homology is simply $\mathbb{F}[v]$. When $t=1$, the complex is $\mathrm{CFK}^{\prime}$ considered above. Define $\Upsilon_{K}(t)$ to be the maximal grading of any $v$-non-torsion element; in particular, $v(K)=\Upsilon_{K}(1)$. Like Hom's homomorphisms, $\Upsilon$ detects $\mathbb{Z}^{\infty}$ direct summands in $\mathscr{C}_{T S}$ [99]; see [70] for an alternative formulation of the invariant $\Upsilon$ and see [7, 26] for further developments.

\section{HeEgAard DiAgRAMS}

To understand knot Floer homology, it is useful to have several possible Heegaard diagrams in hand. The first Heegaard diagram, which we will call the standard diagram for a knot projection, is determined as follows.

5.1. The standard diagram for a knot projection. Fix a knot projection $\mathscr{D}$ for $K$ in $\mathbb{R}^{2}$, together with a distinguished edge adjoining the infinite region in the projection complement. The edge is distinguished by placing a star somewhere on the edge, as shown on the left in Figure 2, We call this data a decorated knot projection of $K$. To a decorated knot projection, we can associate a Heegaard diagram representing $K$, as follows. First, singularize the projection, so that the crossings are actually double-points. Next, take a regular neighborhood of the resulting planar graph, to obtain a handlebody $H$ embedded in $\mathbb{R}^{3} \subset S^{3}$. The regions in the complement of the graph in the plane have two distinguished regions that adjoin the marked edge, one of which is the infinite region in $\mathbb{R}^{2}$. For each bounded region in the graph complement, there is a corresponding $\alpha$-circle. In a neighborhood of each crossing, we associate a $\beta$-circle as pictured in Figure 2 Transverse to the marking on the distinguished edge, we choose also a final $\beta$-circle, again as shown in Figure 2, and place the basepoint $w$ and $z$ on either side of it. Note that $\Sigma$ is oriented as $-\partial H$.

We recall here Kauffman's construction of the Alexander polynomial [46]. 

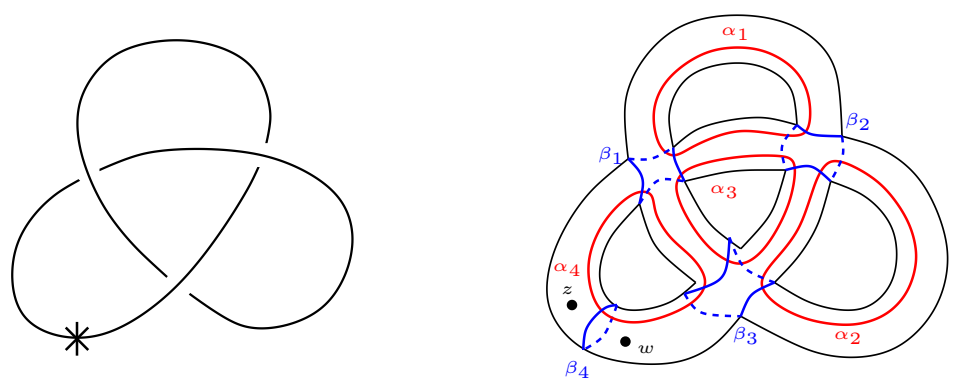

Figure 2. Doubly-pointed Heegaard diagram for the lefthanded trefoil.

Definition 5.1. A Kauffman state for a decorated knot projection of $K$ is a map $\kappa$ that associates to each vertex of $G$ one of the four in-coming quadrants, subject to the following constraints:

- The quadrants assigned by $\kappa$ to distinct vertices are subsets of distinct bounded regions in $\mathbb{R}^{2} \backslash G$.

- The quadrants of the bounded region that meets the distinguished edge are not assigned by $\kappa$ to any of the vertices in $G$.

See Figure 3 for examples.
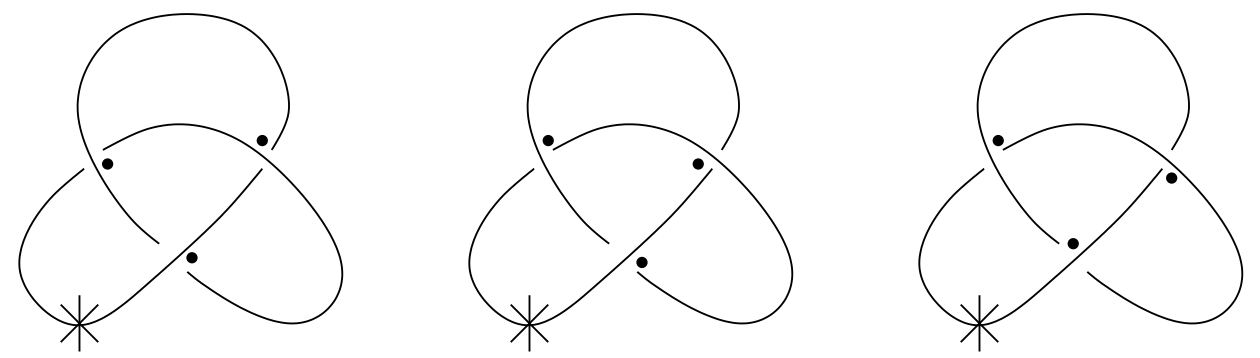

FiguRE 3. Kauffman states for the left-handed trefoil.

Here all three of the states for this projection.

Definition 5.2. Label the four quadrants about each crossing with 0 , and $\pm \frac{1}{2}$, according to the orientations as specified in the first line of Figure 4 . The Alexander function of a Kauffman state $\kappa, A(\kappa)$, is a sum, over each crossing, of the contribution of the quadrant occupied by the state. The Maslov function of a Kauffman state $\kappa$ is obtained similarly, only now the local contributions are as specified in the second line of Figure 4 .

Let $\mathfrak{S}=\mathfrak{S}(\mathscr{D})$ denote the set of Kauffman states. Kauffman shows that the Alexander polynomial is computed by

$$
\Delta_{K}(t)=\sum_{\mathbf{x} \in \mathfrak{S}}(-1)^{M(\mathbf{x})} t^{A(\mathbf{x})} .
$$

(Note that Kauffman does not define $M(\mathbf{x})$, which is not needed for the Alexander polynomial, only its parity.) 

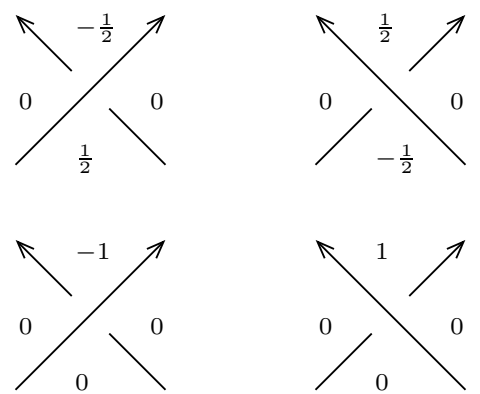

Figure 4. Local Alexander and Maslov contributions. The first row illustrates the local Alexander contributions, and the second the local Maslov contributions of each crossing.

To put Kauffman states in even more familiar territory, recall that a knot projection can be given a checkerboard coloring, coloring each region on the graph complement black and white so that the two regions meeting along each edge are colored differently. There is a planar graph, the "black graph", whose vertices correspond to the black regions in the checkerboard coloring, and whose edges correspond to crossings in the decorated knot diagram. There is a straightforward one-to-one correspondence between maximal subtrees in the black graph and Kauffman states; see [46].

The relevance of Kauffman states to knot Floer homology is the following observation from 88: the Heegaard states in the standard diagram for a knot projection correspond to the Kauffman states of the marked projection, via a correspondence which identifies the corresponding Maslov and Alexander functions.

Although this information is insufficient to compute knot Floer homology, since the differentials count pseudo-holomorphic disks, it does give computations in many cases. For example, an elementary argument shows that for an alternating diagram, $A(\mathbf{x})-M(\mathbf{x})$ is independent of the Kauffman state $\mathbf{x}$. A little more work shows that for an alternating knot, $M(\mathbf{x})-A(\mathbf{x})=\frac{\sigma(K)}{2}$. Theorem 3.3 is an immediate consequence of these considerations. Eun-Soo Lee [61] has shown that a corresponding result also holds for Khovanov homology [48, 3].

5.2. $(1,1)$ diagrams. In knot theory, a knot is said to have a type $(g, b)$ representation if there is a genus $g$ Heegaard splitting for $S^{3}$ in which the knot meets each of the two handlebodies as a union of $b$ standard, unknotted arcs [13]. Thus, the doubly-pointed Heegaard diagrams described above give type $(g, 1)$ representations of knots.

There is a class of knots for which the Heegaard Floer homology particularly easy to compute, which can be represented on the torus, equipped with two basepoints; i.e. which have representations of type $(1,1)$. Explicitly, suppose that $\Sigma$ is a surface of genus 1 , equipped with two basepoints $w$ and $z$, and two curves $\alpha$ and $\beta$ which are isotopic (via an isotopy that crosses $w$ and $z$ ) to two curves $\alpha^{\prime}$ and $\beta^{\prime}$ that meet transversely in a single intersection point. Knots with such representations include all torus knots and all 2-bridge knots (knots on which there is a height function with 4 critical points: 2 maxima and 2 minima); see 8 for a classification. 
For a $(1,1)$ knot, the Heegaard Floer homology takes place in the first symmetric product of the torus $\Sigma$, i.e. within $\Sigma$ itself. Thus, the holomorphic disk counts are combinatorial; see [91, 39.

5.3. Grid diagrams. A Heegaard diagram representing a $(g, b)$ decomposition can be represented by a Heegaard surface $\Sigma$ equipped now with $g+b-1 \alpha$-curves and $g+b-1 \beta$-curves and $2 b$ basepoints $w_{1}, \ldots, w_{b}$ and $z_{1}, \ldots, z_{b}$. The $\alpha$-curves are required to be pairwise disjoint, and to span a half-dimensional subspace of $H_{1}(\Sigma)$; the $\beta$-curves are required to satisfy the same property. By our homological conditions, the surface obtained by cutting $\Sigma$ along the $\alpha$-curves has $b$ connected components. Our diagrams will satisfy the following additional property: each of these connected components is required to have exactly one $w$-basepoint and one $z$-basepoint. Cutting $\Sigma$ along the $\beta$-curves is gives $b$ components, and each component is required to have exactly one $w$-basepoint and one $z$-basepoint. This data specifies a three-manifold $Y$ by the natural generalization of the earlier construction: attach three-dimensional two handles to $[-1,1] \times \Sigma$ along $\{-1\} \times \alpha_{i}$ and $\{1\} \times \beta_{j}$. We are left with a three-manifold whose boundary consists of a collection of two-spheres. Fill each two-sphere with a three-ball.

We can construct an oriented link in $Y$ that meets $\Sigma$ in $\mathbf{w} \cup \mathbf{z}$, by the following construction. In each component of $\Sigma \backslash\left(\alpha_{1} \cup \cdots \cup \alpha_{g+b-1}\right)$, find an arc that connects the corresponding $w_{i}$ and $z_{j}$, and push that arc into $[-1,0] \times \Sigma$, so that it meets $\{0\} \times \Sigma$ exactly at $w_{i}$ and $z_{j}$. Find corresponding arcs in $\Sigma \backslash \boldsymbol{\beta}$, and push those into $[0,1] \times \Sigma$. The two types of basepoints give one-to-one correspondences

$$
f_{\mathbf{w}}: \pi_{0}(\Sigma \backslash \boldsymbol{\alpha}) \rightarrow \pi_{0}(\Sigma \backslash \boldsymbol{\beta}) \quad \text { and } \quad f_{\mathbf{z}}: \pi_{0}(\Sigma \backslash \boldsymbol{\alpha}) \rightarrow \pi_{0}(\Sigma \backslash \boldsymbol{\beta}) ;
$$

so $f_{\mathbf{z}}^{-1} \circ f_{\mathbf{w}}$ is a permutation of $\pi_{0}(\Sigma \backslash \boldsymbol{\alpha})$. That permutation can be written as a product of cycles; and the number of cycles in the description gives the number of components of the resulting link.

Heegaard Floer homology has a generalization to this construction, as well. The ambient symplectic manifold now is $\operatorname{Sym}^{g+b-1}(\Sigma)$, equipped with two $g+b-1$ dimensional tori $\mathbb{T}_{\alpha}$ and $\mathbb{T}_{\beta}$. The chain complex $\mathrm{CFK}^{-}(\mathscr{H})$ now is defined over the polynomial algebra $\mathbb{F}\left[U_{1}, \ldots, U_{b}\right]$, with differential given by

$$
\partial^{-}(\mathbf{x})=\sum_{\mathbf{y} \in \mathfrak{S}} \sum_{\left\{\phi \in \pi_{2}(\mathbf{x}, \mathbf{y}) \mid \mu(\phi)=1, n_{z_{1}}(\phi)=\cdots=n_{z_{b}}(\phi)=0\right\}} \#\left(\frac{\mathcal{M}(\phi)}{\mathbb{R}}\right) U_{1}^{n_{w_{1}}(\phi)} \cdots U_{b}^{n_{w_{b}}(\phi)} \mathbf{y}
$$

When the multiply-pointed Heegaard diagram represents a knot $K$, then all of the $U_{i}$ variables act the same in homology, and the resulting $\mathbb{F}[U]$-module is isomorphic to the bigraded knot Floer homology $\operatorname{HFK}^{-}(K)$ described earlier; cf. [74, 95].

This observation is especially powerful for a particular class of Heegaard diagrams called grid diagrams, where $\Sigma$ has genus 1, all of the $\alpha$-curves are parallel (i.e. isotopic to one another), and all the $\beta$-curves are parallel.

It is a classical result that every knot has such a diagram: indeed, a projection for a knot with $c$ crossings can be turned into a grid diagram for $K$ with $b=$ $c+2 \alpha$-curves and $\beta$-curves. Moreover, these diagrams are also "nice" in the sense introduced by Sucharit Sarkar. Sarkar showed that for certain Heegaard diagrams, the holomorphic disk counts appearing in the Heegaard Floer differential 
have an explicit, topological formulation [109. The key result of Ciprian Manolescu, Sucharit Sarkar, and the second author in [74 states that the holomorphic disk counts appearing in Equation (5.1) for grid diagrams is a combinatorial count of certain embedded rectangles in the Heegaard torus. In [2], these techniques are used to compute the knot Floer homology groups of knots with $\leq 12$ crossings.

The resulting chain complex, whose generators correspond to permutations and whose differential counts embedded rectangles, can be taken as a definition for the theory rather than a computation. Invariance can be formulated and proved within the realm of grid diagrams: there is a well understood set of moves that connect any two grid diagrams representing the same knot [12, 22]. One can construct isomorphisms between the corresponding "grid homology groups", to show that the result is knot invariant. This is the approach taken in [75]; see also [84].

The basic setup of grid homology requires little machinery: gone are the pseudoholomorphic curves, replaced instead by embedded rectangles. This makes the material perhaps more accessible to students trying to enter the subject. The perspective offered by grids naturally points to further applications, especially to Legendrian knot theory [100, 81]; see also [84, Chapter 12]. Moreover, some of the topological applications have proofs purely within the framework of grid diagrams. As pointed out earlier, the slice genus bounds have a combinatorial formulation (see Theorems 4.7 and 4.8 above). Some non-orientable 4 -genus bounds (see Theorem 4.9) have combinatorial proofs [83].

Working entirely in the world of grid diagrams does have some disadvantages, though. At present, many of the topological applications cannot be understood from the grid perspective. More frustratingly, the chain complexes associated to grid diagrams tend to be large and unwieldy. For a knot represented by an $n \times n$ grid diagram, the grid chain complex has $n$ ! generators.

Much has been written on the topic of grid diagrams, so we refer the interested reader to the literature. We will focus instead on a different more algebraic computational approach [98, 86, 85, motivated by "bordered Floer homology" 67].

\section{Bordered Preliminaries}

Bordered Floer homology is an invariant for three-manifolds with boundary introduced in 2008, Robert Lipshitz, Dylan Thurston, and the second author [67, 64]. This theory associates a differential graded algebra to a surface $F$ equipped with a parameterization, $\mathscr{A}(F)$. To an oriented three-manifold $Y_{1}$, equipped with an identification $F \cong \partial Y_{1}$, the bordered theory associates an $\mathscr{A}_{\infty}$ module over this algebra, denoted $\widehat{\mathrm{CFA}}\left(Y_{1}\right)$. For an oriented three-manifold $Y_{2}$ whose boundary is identified with $-\widehat{F}$, the theory associates an algebraic object, called a "type $D$ structure" $\widehat{\mathrm{CFD}}\left(Y_{2}\right)$, over $\mathscr{A}(F)$, which can be thought of as a kind of free differential module over $\mathscr{A}(F)$. The module operations are defined by certain pseudo-holomorphic disks occurring in naturally adapted Heegaard diagrams that represent bordered three-manifolds. We recall here some of the formal aspects of this theory, as they serve as a motivation for some algebraic constructions for knot Floer homology which we will describe later. 

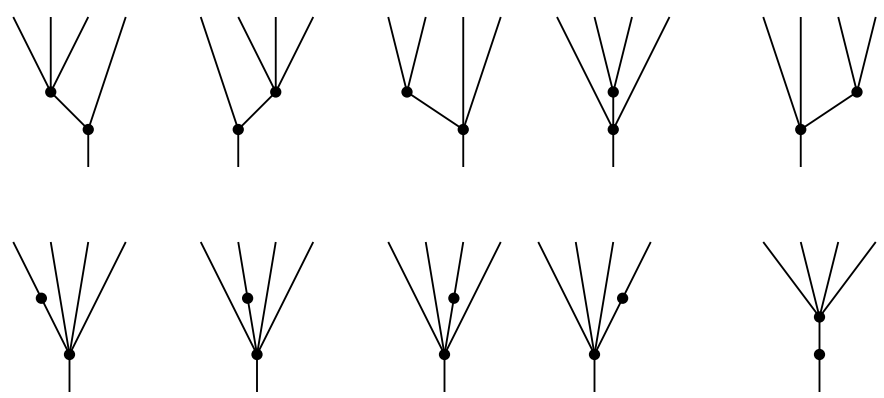

Figure 5 . The $\mathscr{A}_{\infty}$ relation with four inputs. The sum of the operations associated to these trees vanishes; for example, the tree on the top left contributes $\mu_{2}\left(\mu_{3}\left(a_{1}, a_{2}, a_{3}\right), a_{4}\right)$.

As a preliminary point, recall that differential graded algebra $\mathscr{A}$ is a graded vector space $\mathscr{A}$ equipped with an associative multiplication and a differential, which are compatible by the Leibniz rule

$$
d(a \cdot b)=(d a) \cdot b+a \cdot(d b) .
$$

We suppress signs here, as we are working with coefficients in $\mathbb{Z} / 2 \mathbb{Z}$. Sometimes the differential and the multiplication are denoted by the more uniform notation

$$
\mu_{1}: \mathscr{A} \rightarrow \mathscr{A} \quad \text { and } \quad \mu_{2}: \mathscr{A} \otimes \mathscr{A} \rightarrow \mathscr{A} .
$$

Then, the structure relations are $\mu_{1} \circ \mu_{1}=0, \mu_{2}\left(\mu_{2}(a, b), c\right)+\mu_{2}\left(a, \mu_{2}(b, c)\right)=0$ (associativity), and $\mu_{1}\left(\mu_{2}(a, b)\right)=\mu_{2}\left(\mu_{1}(a), b\right)+\mu_{2}\left(a, \mu_{1}(b)\right)$.

Differential graded algebras have a natural generalization to $\mathscr{A}_{\infty}$ algebras [47], which are graded vector spaces $\mathscr{A}$ equipped with a sequence of maps

$$
\left\{\mu_{n}: \mathscr{A}^{\otimes n} \rightarrow \mathscr{A}\right\}_{n=1}^{\infty},
$$

satisfying an infinite sequence of structure relations (generalizing the three structure relations for differential graded algebras stated above), called the $\mathscr{A}_{\infty}$ relations. To state these, it is useful to think of planar trees $T$, with $k$ inputs and one output. Each such tree gives rise to a map $\mu(T): \mathscr{A}^{\otimes k} \rightarrow \mathscr{A}$, where each vertex with valence $d$ is labelled by the operation $\mu_{d-1}$. The $\mathscr{A}_{\infty}$ relation with $k$ inputs, states the sum of $\mu(T)$, taken over all trees $T$ with $k$ inputs and exactly two internal vertices, vanishes. For example, there is a single tree with two internal vertices: it is the linear tree with two valence two vertices. So the $\mathscr{A}_{\infty}$ relation in this case states that $\mu_{1} \circ \mu_{1}=0$. A more interesting example is shown in Figure 5 . From this perspective, a differential graded algebra is an $\mathscr{A}_{\infty}$ algebra with $\mu_{n}=0$ for all $n \geq 3$.

The $\mathscr{A}_{\infty}$ relations can alternatively be formalized as follows. Consider the "bar complex", the vector space

$$
\operatorname{Bar}(\mathscr{A})=\bigoplus_{i=1}^{\infty} \mathscr{A}^{\otimes i}
$$

equipped with the endomorphism

(6.1)

$\bar{\partial}\left(a_{1} \otimes \cdots \otimes a_{n}\right)=\sum_{r \geq 0, s>0, r+s \leq n}\left(a_{1} \otimes \cdots \otimes a_{r}\right) \otimes \mu_{s}\left(a_{r+1} \otimes \cdots \otimes a_{r+s}\right) \otimes\left(a_{r+s+1} \otimes \cdots \otimes a_{n}\right)$. 
The $\mathscr{A}_{\infty}$ relation is equivalent to the condition that $\bar{\partial} \circ \bar{\partial}=0$.

Over a differential graded algebra $\mathscr{A}$, it is natural to consider differential graded modules $M$, which are equipped with a differential $m_{1}: M \rightarrow M$, and an associative action $m_{2}: M \otimes \mathscr{A} \rightarrow M$. These objects have a natural $\mathscr{A}_{\infty}$ generalizations: an $\mathscr{A}_{\infty}$ module $M$ is a graded vector space equipped with a sequence of maps

$$
\left\{m_{n}: M \otimes \mathscr{A}^{\otimes(n-1)} \rightarrow M\right\}_{n=1}^{\infty} .
$$

Again, these are required to satisfy an $\mathscr{A}_{\infty}$ relation, which is exactly as in the case for algebras, with the understanding that now all trees $T$ have a distinguished leftmost strand (corresponding to $M$ ), along which all vertices are labelled with $m_{i}$, rather than $\mu_{i}$ which labels all other vertices.

Stasheff [11] introduced $\mathscr{A}_{\infty}$ algebras in his study of algebraic topology for $H$ spaces. They have since resurfaced in a number of settings: for example, they have taken a central role in symplectic geometry [51, 110; they have found applications in gauge theory [59, 5, 54]; and of course they are also at the heart of bordered Floer homology. Although we will not need $\mathscr{A}_{\infty}$ algebras in our subsequent discussions, we will be considering $\mathscr{A}_{\infty}$ modules over differential graded algebras.

Next, we recall the notation of a $D$ structure over a differential graded algebra $\mathscr{A}$, which is another key player in the bordered theory. A type $D$ structure is a graded vector space $X$, equipped with a map

$$
\delta^{1}: X \rightarrow \mathscr{A} \otimes X
$$

satisfying a structure equation

$$
\left(\mu_{2} \otimes \operatorname{Id}_{X}\right) \circ\left(\delta^{1} \otimes \operatorname{Id}_{X}\right) \circ \delta^{1}+\left(\mu_{1} \otimes \operatorname{Id}_{X}\right) \circ \delta^{1}=0 .
$$

More concretely, if $X$ has a basis $\left\{\mathbf{x}_{i}\right\}_{i=1}^{n}$, we can write

$$
\delta^{1}\left(\mathbf{x}_{i}\right)=\sum_{j=1}^{n} a_{i, j} \otimes \mathbf{x}_{j},
$$

for $a_{i, j} \in \mathscr{A}$. The structure relation takes the form

$$
d a_{i, k}+\sum_{j} a_{i, j} \cdot a_{j, k}=0 .
$$

There is a natural pairing between $\mathscr{A}_{\infty}$ modules $M$ and type $D$ structures $X$ over $\mathscr{A}$ [67], denoted $M \otimes X$, defined as follows. Iterate $\delta^{1}$ to define a map

$$
\delta^{j}: X \rightarrow \mathscr{A}^{\otimes j} \otimes X .
$$

More precisely, define $\delta^{j}$ inductively by $\delta^{0}=\operatorname{Id}_{X}$, and

$$
\delta^{j}=\left(\operatorname{Id}_{\mathscr{A} \otimes(j-1)} \otimes \delta^{1}\right) \circ \delta^{j-1}
$$

for $j>0$; e.g.

$$
\begin{aligned}
& \delta^{2}(x)=\left(\operatorname{Id}_{\mathscr{A}} \otimes \delta^{1}\right) \circ \delta^{1} \\
& \delta^{3}(x)=\left(\operatorname{Id}_{\mathscr{A} \otimes \mathscr{A}} \otimes \delta^{1}\right) \circ\left(\operatorname{Id}_{\mathscr{A}} \otimes \delta^{1}\right) \circ \delta^{1} .
\end{aligned}
$$

Equip the vector space $M \otimes X$ with the endomorphism

$$
D(p \otimes x)=\sum_{j=0}^{\infty}\left(m_{j+1} \otimes \operatorname{Id}_{X}\right) \circ \delta^{j}(x) .
$$


In general, the sum defining $D$ may not be finite; but there are some instances where it is. For example, the module $M$ is said to be algebraically bounded $m_{j}=0$ for all $j$ sufficiently large; and a type $D$ structure $X$ is said to be algebraically bounded if $\delta^{j}=0$ for all $j$ sufficiently large. Boundedness of either structure is sufficient to ensure finite sums in the definition of $D$.

In cases where $D$ is well defined, $D^{2}=0$; i.e. $(M \otimes X, D)$ is a chain complex. This chain complex is denoted $M \otimes X$ and it agrees with the derived tensor product of the two $\mathscr{A}_{\infty}$ modules underlying $M$ and $\mathscr{A} \otimes X$; see [67].

A key property of bordered Floer homology is a pairing theorem, which, for a three-manifold $Y$ decomposed as $Y=Y_{1} \cup_{F} Y_{2}$, expresses $\widehat{\mathrm{HF}}(Y)$ in terms of the above pairing between the type $D$ and the type $A$ structures of the pieces, $\widehat{\mathrm{HF}}(Y) \simeq H\left(\widehat{\mathrm{CFA}}\left(Y_{1}\right) \otimes \widehat{\mathrm{CFD}}\left(Y_{2}\right)\right)$.

Bimodules have a natural generalization to the $\mathscr{A}_{\infty}$ setting. Informally, if $\mathscr{A}_{1}$ and $\mathscr{A}_{2}$ are differential graded algebras, a type $D A$ bimodule ${ }^{\mathscr{A}_{1}} X_{\mathscr{A}_{2}}$ is an object which can be viewed as a type $D$ structure over $\mathscr{A}_{1}$, but it also has higher operations

$$
\delta_{i+1}^{1}: X \otimes \mathscr{A}_{2}^{\otimes i} \rightarrow \mathscr{A}_{1} \otimes X,
$$

satisfying an appropriate $\mathscr{A}_{\infty}$ relation [66].

Bimodules play the following role in the bordered theory. Recall that modules associated to a three manifold depend on the boundary parameterization. To each mapping class $\phi: F \rightarrow F$ there is a corresponding bimodule with the property that if $Y_{1}^{\prime}$ is obtained by composing the boundary parameterization of $Y_{1}$ with $\phi$, then $\widehat{\mathrm{CFD}}\left(Y_{1}^{\prime}\right)$ is the tensor product of $\widehat{\mathrm{CFD}}\left(Y_{1}\right)$ with the associated bimodule; see [66].

Bordered Floer homology can be used to effectively compute $\widehat{\mathrm{HF}}(Y)$ (with $\mathbb{F}$ coefficients). The key point is that the bimodules associated to mapping class group generators can be computed explicitly [65. Thus, if we start from a Heegaard decompositon of $Y$, thought of as a union of two standardly framed handlebodies, glued via an identification $\phi$, which is expressed as a product of the mapping class group generators, then $\widehat{\mathrm{HF}}(Y)$ can be obtained as an iterated tensor product, where the two outermost factors are the modules associated to the standard handlebodies, and the inner factors are the bimodules associated to the mapping class group generators appearing in the factorization of $\phi$. Conversely, this description can be taken as the definition of $\widehat{\mathrm{HF}}(Y)$, and its topological invariance properties can be verified by some model computations. This is the perspective pursued in work of Bohua Zhan [120].

We will describe next an analogous bordered formulation for computing knot Floer homology; compare also [119, 102].

\section{BORDERED ALGEBRAS AND KNOT INVARIANTS}

Bordered knot Floer homology, defined in [98] and [86], is a technique for computing knot Floer homology, which can be thought of as obtained from slicing a decorated knot projection along horizontal slices. Specifically, cut the decorated knot projection into slices $y=t_{1}<\cdots<t_{m}$, with the following properties: 
- the portion of the diagram with $y \leq t_{1}$ consists of a single strand with the global minimum on it

- the portion with $y \geq t_{m}$ consists of a single strand with the global maximum on it.

- each portion of the diagram with $t_{i} \leq y \leq t_{i+1}$ is one of the following three standard pieces: a local maximum, a local minimum, or a crossing.

To each $y=t_{i}$ slice of the diagram, we will associate an algebra. To each standard piece we associate a bimodule over the two algebras associated to its boundary. A chain complex computing the invariant is then obtained by tensoring together all of these bimodules. These generators correspond to Kauffman states; and indeed generators of the intermediate bimodules correspond to certain "partial" Kauffman states. The homology of the resulting chain complex is a knot invariant. We describe these ingredients in a little more detail presently.

7.1. Partial knot diagrams. For generic $t$, a decorated knot projection $\mathscr{D}$ in the $(x, y)$ plane meets the line $y=t$ in $2 n$ transverse points. We will draw our diagram so that the distinguished star is the global minimum $y_{0}$ of the function $y$ restricted to the projection.

The portion of the diagram contained in the half-space in $y \geq t$, for generic $t>y_{0}$, is called a upper knot diagram.

Fix an upper diagram, and suppose that it meets the $y=t$ slice at the $2 n$ points $\{(i, t)\}_{i=1}^{2 n}$. These intersection points divide the $y=t$ line into $2 n+1$ connected components $J_{0}=(-\infty, 1), J_{1}=(1,2), \ldots, J_{2 n-1}=(2 n-1,2 n), J_{2 n}=(2 n, \infty)$. An idempotent state $\mathbf{x}$ is an $n$-element subset of $\{0, \ldots, 2 n\}$.

An upper Kauffman state for an upper diagram $y \geq t$ is a pair $(\kappa, \mathbf{x})$ where $\kappa$ is a function that associates to each crossing in the upper diagram one of the four adjacent quadrants, and $\mathbf{x}$ is an idempotent state for the $y=t$ slice of the diagram, subject to the following constraints:

- The quadrants assigned by $\kappa$ to different crossings are subsets of distinct bounded regions in the upper diagram. (The regions which contain quadrants assigned by $\kappa$ are called occupied.)

- The unbounded region meets none of the intervals in $\mathbf{x}$

- Each bounded, unoccupied bounded region contains exactly one of the intervals appearing in $\mathbf{x}$ on its boundary.

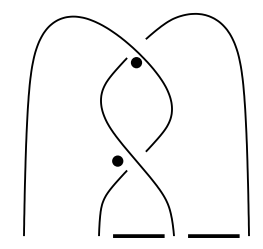

Figure 6. An upper Kauffman state. The black dots represent the quadrants assigned by $\kappa$; the dark intervals on the bottom represent $\mathbf{x}$. This upper diagram has five states. 
Note that any Kauffman state can be restricted to an upper diagram to give an upper Kauffman state.

Upper Kauffman states have the following generalization. A partial knot diagram is a portion of a knot diagram contained in the $(x, y)$ plane with $t_{2} \leq y \leq t_{1}$, so that $t_{1}$ and $t_{2}$ are generic.

A partial Kauffman state is a triple of data $(\kappa, \mathbf{x}, \mathbf{y})$, where $\mathbf{x}$ is a collection of components in the $y=t_{2}$ slice, $\mathbf{y}$ is a collection of components in the $y=t_{1}$ slice, and $\kappa$ is a map that associates to each crossing one of its four adjacent regions, subject to certain constraints.

- Distinct crossings are assigned by $\kappa$ to quadrants contained in distinct regions in the partial diagram.

- If $R$ is occupied, then $\mathbf{y}$ contains all the intervals in $R \cap\left(y=t_{1}\right)$ and $\mathbf{x}$ contains none of the intervals in $R \cap\left(y=t_{2}\right)$.

- If $R$ is unoccupied, then either $R$ meets the $y=t_{1}$ slice, $\mathbf{y}$ contains all but one of the edges of $R \cap\left(y=t_{1}\right)$, and $\mathbf{x}$ contains none of the intervals in $R \cap\left(y=t_{2}\right)$; or $\mathbf{y}$ contains all of the intervals in $R \cap\left(y=t_{1}\right)$ (which now can be empty) and $\mathbf{x}$ contains exactly one of the intervals in the slice $R \cap\left(y=t_{2}\right)$.

Example 7.1. Consider the partial knot diagram consisting of $2 n$ vertical lines. In this partial diagram, the partial Kauffman states $(\kappa, \mathbf{x}, \mathbf{y})$ have $\mathbf{x}=\mathbf{y}$, an arbitrary $n$-element subsets of $\{0, \ldots, 2 n\}$; and $\kappa$ has no information (as there are no crossings).

Example 7.2. Consider the partial knot diagram consisting of $2 n$ vertical lines, and a single additional strand which contains a local maximum, so that two of the strands meet the bottom; see Figure 7 . Assume that the maximum does not appear in the unbounded region. Then, there is a region $R$ in the diagram that meets the top boundary in its $(c-1)^{\text {st }}$ interval, and it meets the bottom boundary its $(c-1)^{s t}$ and $(c+1)^{\text {st }}$ intervals. The partial Kauffman state is then uniquely determined by $\mathbf{x}$, which necessarily contains $c$. There are three cases; a state is said to be of type $\mathbf{X}$ if $\mathbf{x} \cap\{c-1, c, c+1\}=\{c-1, c\}$, it is of type $\mathbf{Y}$ if $\mathbf{x} \cap\{c-1, c, c+1\}=\{c, c+1\}$, and $\mathbf{Z}$ if $\mathbf{x} \cap\{c-1, c, c+1\}=\{c\}$. See Figure $\mathbf{7}$

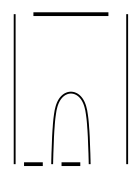

Type $\mathbf{X}$

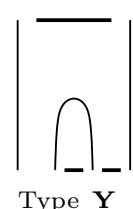

Type $\mathbf{Y}$

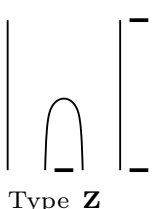

Type $\mathbf{Z}$

Figure 7. Partial Kauffman states for the local maximum. We have drawn here three partial Kauffman states, one of each type.

Example 7.3. Consider the partial knot diagram consisting of $2 n$ strands drawn so that the $i^{\text {th }}$ and $(i+1)^{\text {st }}$ cross exactly once. There are four kinds of partial Kauffman states, according to which of the four regions is assigned to the crossing: $\mathbf{N}, \mathbf{S}, \mathbf{E}$, or $\mathbf{W}$. For the crossing of type $\mathbf{N}, i \in \mathbf{x} \cap \mathbf{y}$ and $\mathbf{x}=\mathbf{y}$; for a crossing 
of type $\mathbf{S}, i \notin \mathbf{x} \cup \mathbf{y}$ and $\mathbf{x}=\mathbf{y}$; for a crossing of type $\mathbf{W}, i-1 \in \mathbf{y}, i \notin \mathbf{y}$, $i-1 \notin \mathbf{x}$ and $i \in \mathbf{x}$, and $\mathbf{y} \backslash\{i-1\}=\mathbf{x} \backslash\{i\}$; for a crossing of type $\mathbf{E}, i+1 \in \mathbf{y}$, $i \notin \mathbf{y}, i+1 \notin \mathbf{x}, i \in \mathbf{x}$, and $\mathbf{y} \backslash\{i+1\}=\mathbf{x} \backslash\{i\}$.

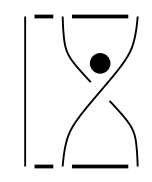

Type $\mathbf{N}$

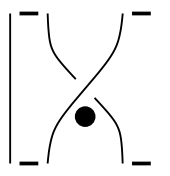

Type $\mathbf{S}$

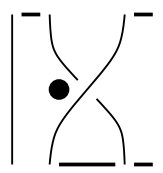

Type $\mathbf{W}$

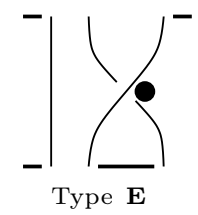

Figure 8. Partial Kauffman states for crossings. We have drawn here three four Kauffman states, one of each type.

7.2. Algebras. We explain how to associate an algebra to each horizontal slice of a knot diagram. The horizontal slice can be thought of as a collection of $2 n$ points on the real line. The portion of the knot projection above this horizontal slice gives a pairing between the $2 n$ points. Specifically, if we slice the projection at the line $y=t$, so that the knot meets the line in points $\{(i, t)\}_{i=1}^{2 n}$, then $i$ and $j$ are matched if $(i, t)$ and $(j, t)$ are joined by an arc in the diagram contained in the portion of the diagram where $y \geq t$. We denote this data by $M$. We will now define the corresponding algebra $\mathscr{A}(n, M)$.

As a preliminary step, we define an algebra $\mathscr{B}_{0}(m, k)$ associated to $m$ points $\{1, \ldots, m\}$ and an integer $0 \leq k \leq m+1$; see [98. The algebra is defined over the polynomial algebra $\mathbb{F}\left[U_{1}, \ldots, U_{m}\right]$; and it is is equipped with a set of preferred idempotents, which correspond to $k$ element subsets of $\{0, \ldots, m\}$ or, equivalently, increasing functions $\mathbf{x}:\{1, \ldots, k\} \rightarrow\{0, \ldots, m\}$ called idempotent states. Let $I(\mathbf{x})$ denote the idempotent corresponding to the idempotent state.

Given any idempotent states $\mathbf{x}, \mathbf{y}$, the $\mathbb{F}\left[U_{1}, \ldots, U_{m}\right]$-module $I(\mathbf{x}) \cdot \mathscr{B}_{0}(m, k) \cdot I(\mathbf{y})$ is isomorphic to $\mathbb{F}\left[U_{1}, \ldots, U_{m}\right]$;i.e. it is given with a preferred generator, which we denote $\gamma_{\mathbf{x}, \mathbf{y}}$.

Thus, for any $\mathbf{x}, \mathbf{y}, \mathbf{z}, \gamma_{\mathbf{x}, \mathbf{y}} \cdot \gamma_{\mathbf{y}, \mathbf{z}}=P_{\mathbf{x}, \mathbf{y}, \mathbf{z}} \cdot \gamma_{\mathbf{x}, \mathbf{z}}$ for some $P_{\mathbf{x}, \mathbf{y}, \mathbf{z}} \in \mathbb{F}\left[U_{1}, \ldots, U_{m}\right]$. To specify the multiplication on $\mathscr{B}_{0}(m, k)$, it suffices to specify the $P_{\mathbf{x}, \mathbf{y}, \mathbf{z}}$ for all triples of idempotent states, which we do as follows. Each idempotent state $\mathbf{x}$ has a weight vector $v^{\mathbf{x}} \in \mathbb{Z}^{m}$, with components given by

$$
v_{i}^{\mathbf{x}}=\#\{x \in \mathbf{x} \mid x \geq i\} .
$$

Let $P_{\mathbf{x}, \mathbf{y}, \mathbf{z}}$ to be the monomial in $\mathbb{F}\left[U_{1}, \ldots, U_{m}\right], U_{1}^{n_{1}} \cdots U_{m}^{n_{m}}$, where $n_{i}$ is given by

$$
n_{i}=\frac{1}{2}\left(\left|v_{i}^{\mathbf{x}}-v_{i}^{\mathbf{y}}\right|+\left|v_{i}^{\mathbf{y}}-v_{i}^{\mathbf{z}}\right|-\left|v_{i}^{\mathbf{x}}-v_{i}^{\mathbf{z}}\right|\right)
$$

Let $L_{i} \in \mathscr{B}_{0}$ with $i \in\{1, \ldots, m\}$ be the sum of the generators $\gamma_{\mathbf{x}, \mathbf{y}} \in I_{\mathbf{x}} \cdot \mathscr{B}_{0} \cdot I_{\mathbf{y}}$ taken over all pairs of idempotent states $\mathbf{x}$ and $\mathbf{y}$ with the property that

$$
v_{j}^{\mathbf{x}}-v_{j}^{\mathbf{y}}= \begin{cases}1 & \text { if } i=j \\ 0 & \text { otherwise. }\end{cases}
$$

Similarly, define $R_{i}$ to be the sum of all the elements $\gamma_{\mathbf{y}, \mathbf{x}} \in I_{\mathbf{y}} \cdot \mathscr{B}_{0} \cdot I_{\mathbf{x}}$, where $\mathbf{x}$ and $\mathbf{y}$ run over all idempotent states as above. 
Let $\mathscr{B}(m, k)$ be the quotient algebra of $\mathscr{B}_{0}(m, k)$ by the relations

$$
L_{i+1} \cdot L_{i}=0, \quad R_{i} \cdot R_{i+1}=0
$$

and

$$
I_{\mathbf{x}} \cdot U_{j}=0
$$

if $\mathbf{x}^{-1}(\{j-1, j\})=\emptyset$; i.e. if $\mathscr{I}$ denotes the two-sided ideal generated by $L_{i+1} \cdot L_{i}$, $R_{i} \cdot R_{i+1}$, and $\mathbf{I}_{\mathbf{x}} \cdot U_{j}$ as above, then $\mathscr{B}=\mathscr{B}_{0} / \mathscr{I}$.

We define a differential graded algebra $\mathscr{A}(n, M)$ by adjoining $n$ central elements $C_{i, j}$ to $\mathscr{B}(2 n, n)$, one for each $\{i, j\} \in M$, which satisfy the relation $C_{i, j}^{2}=0$. We introduce a differential $d$ on $\mathscr{A}(n, M)$ by $d C_{i, j}=U_{i} U_{j}$.

The algebras can be given gradings, after choosing an orientation on $K$; see [86].

Example 7.4. The algebra $\mathscr{B}(2,1)$ has the following geometric description. Consider the graph with three vertices, labelled 0,1 , and 2 , and two edges, one connecting 0 to 1 and another connecting 1 and 2. Think of the path as drawn horizontally, so that 1 is to the right of 0 . The algebra $\mathscr{B}(2,1)$ can be thought of as the quotient of the algebra of all paths in this graph, and obtained by dividing out by all paths that connect 0 and 2. The constant paths at 0,1 , and 2 correspond to the three idempotents $\mathbf{I}_{\{0\}}, \mathbf{I}_{\{1\}}$, and $\mathbf{I}_{\{2\}}$; the first edge corresponds to $L_{1}$ and $R_{1}$ (depending on the direction it is crossed), and the second corresponds to $L_{2}$ and $R_{2}$. Here, $U_{1}=L_{1} \cdot R_{1}+R_{1} \cdot L_{1}$ and $U_{2}=L_{2} \cdot R_{2}+R_{2} \cdot L_{2}$. Clearly, the relation $U_{1} U_{2}=0$ holds in $\mathscr{B}(2,1)$.

To construct $\mathscr{A}(1,\{1,2\})$, we adjoin one variable $C_{\{1,2\}}$ whose square is zero. We think of this as differential graded algebra, but the differential is identically zero. In particular,

$$
\mathbf{I}_{\{1\}} \cdot \mathscr{A}(1,\{1,2\}) \cdot \mathbf{I}_{\{1\}} \cong \frac{\mathbb{F}\left[U_{1}, U_{2}, C_{\{1,2\}}\right]}{\left(U_{1} U_{2}, C_{\{1,2\}}^{2}\right)} .
$$

We will view $\mathscr{A}=\mathscr{A}(n, M)$ as an algebra over the base ring of idempotents

$$
\left.\mathbf{I}(\mathscr{A}) \cong \mathbb{F}^{(2 n+1}{ }_{n}\right) .
$$

As such, an $\mathscr{A}_{\infty}$ module over $\mathscr{A}$ will be a right module over $\mathbf{I}(\mathscr{A})$, and the actions $m_{n}$ will be multilinear over $\mathbf{I}(\mathscr{A})$ : i.e. the tensor products in Equation (6.2) are taken over that ring. Similarly, a type $D$ structure is a left module over $\mathbf{I}(\mathscr{A})$, the map $\delta^{1}$ is a $\mathbf{I}(\mathscr{A})$-module map, and the tensor product appearing in Equation (6.3) is also over that ring. Moreover, in the definition of $M \otimes X$, the underlying vector space is $M \otimes_{\mathbf{I}(\mathscr{A})} X$.

7.3. Bimodules. Having defined the algebra, we must associate bimodules to the various pieces.

First, consider the module associated to the global maximum. Here, there is a single upper Kauffman state, and the corresponding generator $\mathbf{z}$ has

$$
\begin{aligned}
\mathbf{z} & =\mathbf{I}_{\{1\}} \cdot \mathbf{z} \\
\delta^{1} \mathbf{z} & =C_{1,2} \otimes \mathbf{z} .
\end{aligned}
$$

We can think of the tensor products in the construction of $\mathscr{C}(\mathscr{D})$ as an iterative procedure, starting with this type $D$ structure as a first step, and then successively 
increasing the size of the diagram covered by tensoring the type $D$ structure in hand with the DA structure associated to the partial knot diagram immediately below it. As we will indicate below, the generators of this type $D$ structure correspond to upper Kauffman states $(\kappa, \mathbf{x})$ for the diagram, and whose left idempotent is $\mathbf{I}_{\mathbf{x}}$.

Thus, a key ingredient going into this definition is the type $D A$ bimodule associated to each standard partial diagram.

We do not describe the bimodules explicitly here; we refer the interested reader to 86. Instead, we explain how to specify them uniquely up to homotopy equivalence.

To this end, it is useful to make the following observations. Consider the dual complex for the bar complex, i.e.

$$
\operatorname{Cobar}(\mathscr{A})=\bigoplus_{i=1}^{\infty} \operatorname{Hom}\left(\mathscr{A}^{\otimes i}, \mathbb{F}\right)
$$

equipped with a differential which is hom dual to $\bar{\partial}$ as given in Equation (6.1). This is an algebra, with multiplication induced by the natural map

$$
\operatorname{Hom}\left(\mathscr{A}^{\otimes i}, \mathbb{F}\right) \otimes \operatorname{Hom}\left(\mathscr{A}^{\otimes j}, \mathbb{F}\right) \rightarrow \operatorname{Hom}\left(\mathscr{A}^{\otimes(i+j)}, \mathbb{F}\right) .
$$

A bounded $\mathscr{A}_{\infty}$ module over a differential graded algebra $\mathscr{A}$ is the same thing as a type $D$ structure over $\operatorname{Cobar}(\mathscr{A})$. More generally, a (bounded) $D A$ bimodule, $\mathscr{A}_{1} X_{\mathscr{A}_{2}}$ is the same thing as a type $D$ structure over the tensor product algebra $\mathscr{A}_{1} \otimes \operatorname{Cobar}\left(\mathscr{A}_{2}\right)$.

The algebra Cobar tends to be rather large; so instead, it is often convenient to find a smaller quasi-isomorphic version $\mathscr{A}^{\prime}$. Then, up to quasi-isomorphism, a bounded $\mathscr{A}_{\infty}$ module over $\mathscr{A}$ is equivalent to a type $D$ structure over $\mathscr{A}^{\prime}$. (This equivalence is called Koszul duality [63]; see also [103].) Similarly, a suitably bounded type $D A$ bimodule ${ }^{\mathscr{A}_{1}} X_{\mathscr{A}_{2}}$ is uniquely determined (up to quasi-isomorphism) by a corresponding module $\mathscr{A}_{1} \otimes \mathscr{A}_{2}^{\prime} Y$. Correspondingly, type $D$ structures over $\mathscr{A}_{1} \otimes \mathscr{A}_{2}^{\prime}$ are called type DD bimodules over $\mathscr{A}_{1}$ and $\mathscr{A}_{2}^{\prime}$.

There is a handy Koszul dual algebra to $\mathscr{A}(n, M)$, denoted $\mathscr{A}^{\prime}(n, M)$, defined as follows. This is defined over the base algebra $\mathscr{B}(2 n, n+1)$, only now we adjoin $2 n$ variables $E_{1}, \ldots, E_{2 n}$ which satisfy the following relations: $E_{i} \cdot E_{j}=E_{j} \cdot E_{i}$ if $i$ and $j$ are not matched, and $d E_{i}=U_{i}$.

The identity map from $\mathscr{A}(n, M)$ to itself can be thought of as a type $D A$ bimodule over $\mathscr{A}(n, M)$; which we can think of as the bimodule associated to the trivial diagram from Example (7.1). This is Koszul dual to the type $D$ structure $\mathscr{K}$ over $\mathscr{A}(n, M) \otimes \mathscr{A}^{\prime}(n, M)$ whose generators are $\mathbf{I}_{\mathbf{x}} \otimes \mathbf{I}_{\mathbf{y}}$, where $\mathbf{x}$ and $\mathbf{y}$ are complementary idempotent states; i.e. $\mathbf{x} \cup \mathbf{y}=\{0, \ldots, 2 n\}$. The differential is specified by the element

$$
A=\sum_{i=1}^{2 n}\left(L_{i} \otimes R_{i}+R_{i} \otimes L_{i}\right)+\sum_{i=1}^{2 n} U_{i} \otimes E_{i}+\sum_{\{i, j\} \in P} C_{\{i, j\}} \otimes \llbracket E_{i}, E_{j} \rrbracket \in \mathscr{A} \otimes \mathscr{A}^{\prime},
$$

where $\llbracket E_{i}, E_{j} \rrbracket=E_{i} \cdot E_{j}+E_{j} \cdot E_{i}$. Specifically,

$$
\delta^{1}: \mathscr{K} \rightarrow\left(\mathscr{A} \otimes \mathscr{A}^{\prime}\right) \otimes_{\mathbf{I}(\mathscr{A}) \otimes \mathbf{I}\left(\mathscr{A}^{\prime}\right)} \mathscr{K}
$$


is given by $\delta^{1}(v)=A \otimes v$

7.3.1. Crossings. We characterize the type $D A$ bimodule of a positive crossing $\mathscr{A}_{2} \mathscr{P}_{\mathscr{A}_{1}}$, where $\mathscr{A}_{2}=\mathscr{A}\left(n, M_{2}\right), \mathscr{A}_{1}=\mathscr{A}\left(n, M_{1}\right)$, and $M_{1}$ is obtained from $M_{2}$ by composing with the transposition switching $i$ and $i+1$. Its corresponding type $D D$ bimodule $\mathscr{A}_{2}, \mathscr{A}_{1}^{\prime} \mathscr{P}$ is generated by partial Kauffman states with the understanding that left multiplication by $\mathbf{I}_{\mathbf{x}} \otimes \mathbf{I}_{\{0, \ldots, 2 n\} \backslash \mathbf{y}}$ preserves the generator corresponding to $(\kappa, \mathbf{x}, \mathbf{y})$.

Decomposing the partial Kauffman states by type as explained in Example 7.3, the differential has the following types of terms:

$(\mathrm{P}-1) R_{j} \otimes L_{j}$ and $L_{j} \otimes R_{j}$ for all $j \in\{1, \ldots, 2 n\} \backslash\{i, i+1\}$; these connect generators of the same type.

(P-2) $U_{j} \otimes E_{\tau(j)}$ for all $j=1, \ldots, 2 n$

(P-3) $C_{\{\alpha, \beta\}} \otimes\left[E_{\tau(\alpha)}, E_{\tau(\beta)}\right]$, for all $\{\alpha, \beta\} \in M_{2}$; these connect generators of the same type

(P-4) Terms in the diagram below that connect generators of different types:

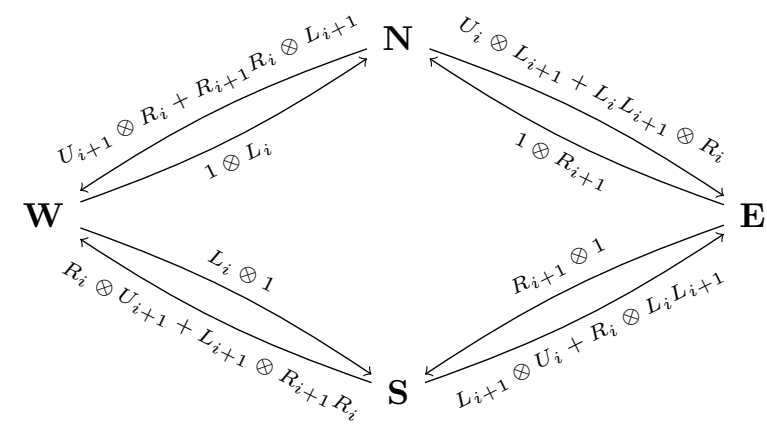

For example, these give rise to terms $\left(1 \otimes R_{i}\right) \otimes \mathbf{W}+\left(1 \otimes L_{i+1}\right) \otimes \mathbf{E}$ in $\delta^{1}(\mathbf{N})$.

The negative crossing works similarly, except that Equation (7.1) is replaced by:

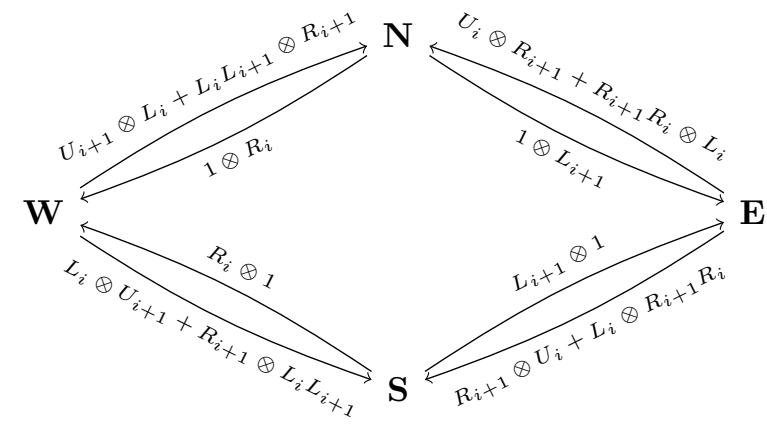

7.3.2. Local maximum. Consider the partial knot diagram of a local maximum from Example 7.2. The type $D A$ bimodule of this partial knot diagram ${ }^{A_{2}} \Omega_{\mathscr{A}_{1}}$ is defined 
over algebras $\mathscr{A}_{1}$ and $\mathscr{A}_{2}$, and it is specified as follows. Let $\phi_{c}:\{1, \ldots, 2 n\} \rightarrow$ $\{1, \ldots, 2 n+2\}$ be the map

$$
\phi_{c}(j)= \begin{cases}j & \text { if } j<c \\ j+2 & \text { if } j \geq c .\end{cases}
$$

Then,

$$
\mathscr{A}_{1}=\mathscr{A}\left(n, M_{1}\right) \quad \text { and } \quad \mathscr{A}_{2}=\mathscr{A}\left(n+1, \phi_{c}\left(M_{1}\right) \cup\{c, c+1\}\right)
$$

We specify this bimodule up to quasi-isomorphism by defining its dual type $D D$ bimodule $\mathscr{A}_{2}, \mathscr{A}_{1}^{\prime} \Omega$. The generators correspond to partial Kauffman states for the partial knot diagram, again with the convention that $\mathbf{I}_{\mathbf{x}} \otimes \mathbf{I}_{\{0, \ldots, 2 n\} \backslash \mathbf{y}}$ preserves the generator corresponding to $(\kappa, \mathbf{x}, \mathbf{y})$.

The differential is specified by the algebra element $A \in \mathscr{A}_{2} \otimes \mathscr{A}_{1}^{\prime}$

$$
\begin{gathered}
A=\left(L_{c} L_{c+1} \otimes 1\right)+\left(R_{c+1} R_{c} \otimes 1\right)+\sum_{i=1}^{2 n} L_{\phi(i)} \otimes R_{i}+R_{\phi(i)} \otimes L_{i} \\
+C_{\{c, c+1\}} \otimes 1+\sum_{i=1}^{2 n} U_{\phi(i)} \otimes E_{i}+\sum_{\{i, j\} \in M} C_{\{\phi(i), \phi(j)\}} \otimes \llbracket E_{i}, E_{j} \rrbracket
\end{gathered}
$$

where we have dropped the subscript $c$ from $\phi_{c}=\phi$.

In more detail, decomposing partial Kauffman states according to the type $\mathbf{X}, \mathbf{Y}$, and $\mathbf{Z}$ specified in Example 7.2, the differential on the bimodule has terms are of the following types:

$(\Omega-1) R_{\phi(j)} \otimes L_{j}$ and $L_{\phi(j)} \otimes R_{j}$ for all $j \in\{1, \ldots, 2 n\} \backslash\{c-1, c\}$; these connect generators of the same type.

$(\Omega-2) U_{\phi(i)} \otimes E_{i}$ for $i=1, \ldots, 2 n$

$(\Omega-3) C_{\{\phi(i), \phi(j)\}} \otimes \llbracket E_{i}, E_{j} \rrbracket$ for all $\{i, j\} \in P$;

$(\Omega-4) C_{\{c, c+1\}} \otimes 1$

$(\Omega-5)$ Terms in the diagram below connect generators of different types.

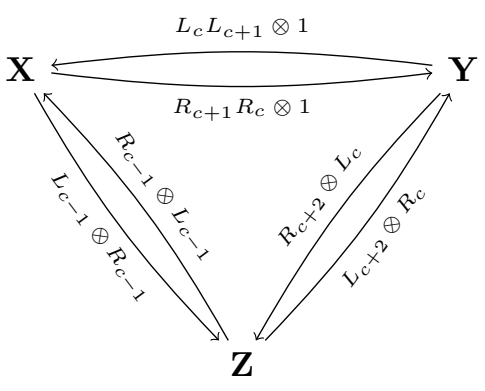

The above description can be readily specialized to the case where the maximum appears in an unbounded region. In these cases, there is only one generator type, $\mathbf{Z}$.

7.3.3. Local minimum. Turning the above example on its top, we have ${ }^{\mathscr{A}_{1}} \mho_{\mathscr{A}_{2}}^{c}$, where $\mathscr{A}_{1}$ and $\mathscr{A}_{2}$ are as in Equation (7.4). We specify this module by describing its dual type $D D$ bimodule $\mathscr{A}_{1}, \mathscr{A}_{2}^{\prime} \mho_{c}$. Its generators are partial Kauffman states, 
with the understanding now that $\mathbf{I}_{\mathbf{x}} \otimes \mathbf{I}_{\{0, \ldots, 2 n+2\} \backslash \mathbf{y}}$ preserves the generator corresponding to $(\kappa, \mathbf{x}, \mathbf{y})$. The $D D$ bimodule is specified by the algebra element $A \in \mathscr{A}_{1} \otimes \mathscr{A}_{2}^{\prime}$

$$
\begin{aligned}
A & =\left(1 \otimes L_{c} L_{c+1}\right)+\left(1 \otimes R_{c+1} R_{c}\right)+\sum_{j=1}^{2 n} R_{j} \otimes L_{\phi(j)}+L_{j} \otimes R_{\phi(j)}+U_{j} \otimes E_{\phi(j)} \\
& +1 \otimes E_{c} U_{c+1}+U_{\alpha} \otimes \llbracket E_{\phi(\alpha)}, E_{c} \rrbracket E_{c+1}+C_{\{\alpha, \beta\}} \otimes \llbracket E_{\phi(\alpha)}, E_{c} \rrbracket \llbracket E_{c+1}, E_{\phi(\beta)} \rrbracket .
\end{aligned}
$$

7.3.4. Global minimum. When we have covered the entire diagram, save for the last piece (the global minimum), we have a type $D$ structure $C$ over the algebra

$$
\mathbf{I}_{\{1\}} \cdot \mathscr{A}(2,1,\{1,1\}) \cdot \mathbf{I}_{\{1\}} \cdot
$$

After dividing out by $C_{\{1,2\}}$, what remains can be thought of as a chain complex over $\mathbb{F}\left[U_{1}, U_{2}\right] / U_{1} U_{2}$. Its homology is the invariant $H(K)$. Dividing out the complex by $U_{2}$ and taking homology gives $H^{-}(K)$; and dividing out by both $U_{1}$ and $U_{2}$ and taking homology gives $\widehat{H}(K)$.

7.4. Topological invariance. It is proved in [86] that the bigraded homology modules $\widehat{H}(\mathscr{D}), H^{-}(\mathscr{D})$ and $H(\mathscr{D})$ are invariants of the underlying oriented knot $K$ represented by the diagram $\mathscr{D}$. This involves checking that the homology of the chain complex is invariant under Reidemeister moves. These relations are proved locally on the level of bimodules.

For example, the bimodules of a crossing satisfy the "braid relations" for any $1 \leq$ $i, j \leq 2 n-1$ : for $|i-j|>1$, these relations give quasi-isomorphisms of bimodules

$$
\mathscr{P}^{j} \otimes \mathscr{P}^{i} \simeq \mathscr{P}^{i} \otimes \mathscr{P}^{j}
$$

(where we have suppressed the algebras which come naturally from the pictures) while if $j=i+1$, then,

$$
\mathscr{P}^{i} \otimes \mathscr{P}^{i+1} \otimes \mathscr{P}^{i} \simeq \mathscr{P}^{i+1} \otimes \mathscr{P}^{i} \otimes \mathscr{P}^{i+1} .
$$

(again for suitably chosen algebras). Thus, we can think of these bimodules as giving a braid group action on the derived category of modules over $\mathscr{A}(n, M)$; compare [50, 66, 71].

The knot invariants $\widehat{H}(K), H^{-}(K)$, and $H(K)$ are designed to agree with their knot Floer homological analogues. One can nonetheless, study them independently of holomorphic methods. For example, one can verify certain fundamental properties within the algebraic realm: relating their graded Euler characteristics with the Alexander polynomial of $K$, establishing a Künneth formula for connected sums, and verifying an algebraic structure result for $\mathrm{H}^{-}(K)$ analogous to Proposition 3.4. see [86].

\section{BORDERED KNOT ALGEBRAS AND PSEUDO-HOLOMORPHIC CURVES}

In fact, we prove that this bordered invariant is equivalent to knot Floer homology [85]. To establish the link between the algebraic constructions and knot Floer homology, it is useful to give a pseudo-holomorphic interpretation of these structures. 
Upper knot diagrams can be represented by suitably decorated (partial) Heegaard diagrams. An upper Heegaard diagram is a surface $\Sigma$ of genus $g$ and $2 n$ boundary components, labelled $Z_{1}, \ldots, Z_{2 n}$, together with the following additional data:

- A collection of disjoint, embedded arcs $\left\{\alpha_{i}\right\}_{i=1}^{2 n-1}$, so that $\alpha_{i}$ connects $Z_{i}$ to $Z_{i+1}$.

- A collection of disjoint embedded closed curves $\left\{\alpha_{i}^{c}\right\}_{i=1}^{g}$ (which are also disjoint from $\left.\alpha_{1}, \ldots, \alpha_{2 n-1}\right)$.

- A collection of embedded, mutually disjoint closed curves $\left\{\beta_{i}\right\}_{i=1}^{g+n-1}$.

Both sets of $\alpha$-and the $\beta$-circles are required to consist of homologically linearly independent curves, and the $\beta$-circles are further required to have the following combinatorial property: the surface obtained by cutting $\Sigma$ along $\beta_{1}, \ldots, \beta_{g+n-1}$, which has $n$ connected components, is required to contain exactly two boundary circles in each component. This requirement gives a matching $M$ on $\{1, \ldots, 2 n\}$ (a partition into two-element subsets), where $\{i, j\} \in M$ if $Z_{i}$ and $Z_{j}$ can be connected by a path that does not cross any $\beta_{k}$.

We sometimes abbreviate the data

$$
\mathscr{H}^{\wedge}=\left(\Sigma, Z_{1}, \ldots, Z_{2 n},\left\{\alpha_{1}, \ldots, \alpha_{2 n-1}\right\},\left\{\alpha_{1}^{c}, \ldots, \alpha_{g}^{c}\right\},\left\{\beta_{1}, \ldots, \beta_{g+n-1}\right\}\right),
$$

and let $M\left(\mathscr{H}^{\wedge}\right)$ be the induced matching.

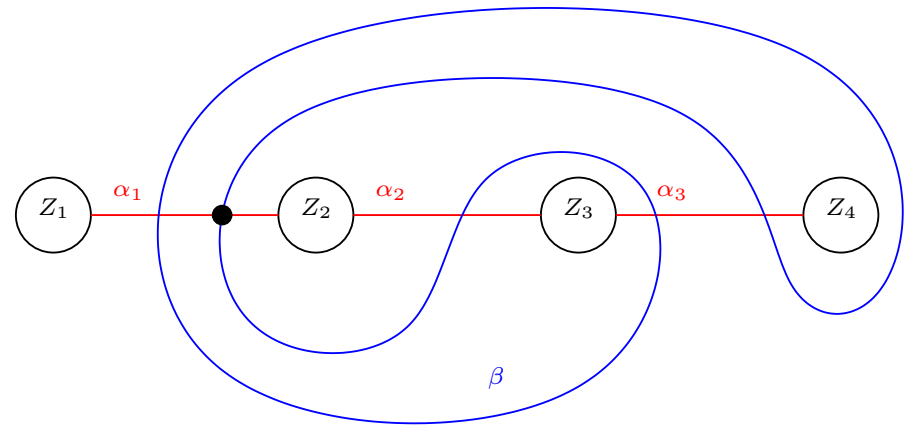

Figure 9. Upper Heegaard diagram. The diagram here is the upper Heegaard diagram for the upper planar diagram from Figure [6] the black dot represents a Heegaard state corresponding to the Kauffman state from Figure 6.

An upper Heegaard state is an $g+n-1$-tuple of points $\mathbf{x}$, each of which is in $\alpha_{i} \cap \beta_{j}$ or $\alpha_{i}^{c} \cap \beta_{j}$ for various $i$ and $j$, so that each circle $\alpha_{i}$ contains an element in $\mathbf{x}$, each $\beta_{j}$ contains an element in $\mathbf{x}$, and no more than one element in $\mathbf{x}$ is contained on each $\alpha$-arc $\alpha_{i}^{c}$. Each Heegaard state $\mathbf{x}$ determines a subset $s(\mathbf{x})$ of $\{1, \ldots, 2 n\}$ with cardinality $n$, or, equivalently, an idempotent $\mathbf{I}_{s(\mathbf{x})}$ in $\mathscr{A}(n, M)$ :

$$
s(\mathbf{x})=\{1, \ldots, 2 n-1\} \backslash\left\{1 \leq i \leq 2 n-1 \mid \mathbf{x} \cap \alpha_{i} \text { is non-empty }\right\} .
$$

Fill in each boundary component $Z_{i}$, to obtain a closed Riemann surface $\bar{\Sigma}$, with $2 n$ marked points $z_{i}$. Extend $\alpha_{i}$ into $\bar{\Sigma}$ to obtain a curve $\bar{\alpha}_{i}$ with $\partial \bar{\alpha}_{i}=z_{i}-z_{i-1}$, as shown in Figure 10. We will place a pair of points $r_{i}$ and $\ell_{i}$ in a neighborhood of $z_{i}$, separated by $\bar{\alpha}_{i-1} \cup \bar{\alpha}_{i}$. (In the special case where $i=1$ and $2 n$, the two 

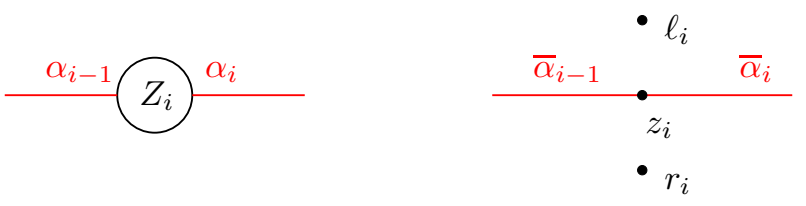

Figure 10. Boundary markings. On the left, we have shown a neighborhood of a boundary component $Z_{i}$ of $\Sigma$. To the right, we have filled in $Z_{i}$, replacing it with the point $z_{i}$.

points $r_{i}$ and $\ell_{i}$ are not separated by this neighborhood, as one of $\alpha_{i-1}$ or $\alpha_{i}$ does not exist.) We will be working with holomorphic disks in $\operatorname{Sym}^{g+n-1}(\Sigma)$, relative to $\mathbb{T}_{\beta}$ and

$$
L_{\alpha}^{0}=\alpha_{1}^{c} \times \cdots \times \alpha_{g}^{c} \times \operatorname{Sym}^{n-1}\left(\bar{\alpha}_{1} \cup \cdots \cup \bar{\alpha}_{2 n-1}\right) .
$$

Note that $L_{\alpha}^{0}$ is a singular space, with singularities contained in the locus where two points are contained on the same $\alpha_{i}$-curve. We will work away from this locus, in the subspace $L_{\alpha} \subset L_{\alpha}^{0}$ consisting of those $n$-1-tuples where no two points lie on the same $\alpha_{i}$ (this corresponds to the "boundary monotonicity" condition of [67]), and each point lies in the interior of some $\alpha_{i}$, denoted $\alpha_{i}^{\circ}$. Clearly, $L_{\alpha}$ is disconnected; in fact

$$
L_{\alpha}=\alpha_{1}^{c} \times \cdots \times \alpha_{g}^{c} \times\left(\bigcup_{\left\{t_{1}, \ldots, t_{n-1}\right\} \subset\{1, \ldots, 2 n-1\}} \alpha_{t_{1}}^{\circ} \times \cdots \times \alpha_{t_{n-1}}^{\circ}\right) .
$$

The upper Heegaard states correspond to intersection points of $L_{\alpha}$ with $\mathbb{T}_{\beta}$. If $\mathbf{x}$ is in the component of $L_{\alpha}$ specified by $\left\{t_{1}, \ldots, t_{n-1}\right\}$, then $\mathbf{I}_{s(\mathbf{x})} \cdot \mathbf{x}=\mathbf{x}$ where $s(\mathbf{x})=\{1, \ldots, 2 n-1\} \backslash\left\{t_{1}, \ldots, t_{n-1}\right\}$.

Let $\pi_{2}(\mathbf{x}, \mathbf{y})$ denote the space of homotopy classes of Whitney disk as before, only now half of the boundary of the disk is mapped into $L_{\alpha}^{0}$, and the other half into $\mathbb{T}_{\beta}$. (In fact, the disks of interest to us will have half their boundary mapped into the closure of $L_{\alpha}, \bar{L}_{\alpha} \subset L_{\alpha}^{0}$.) Each $\phi \in \pi_{2}(\mathbf{x}, \mathbf{y})$ with non-negative local multiplicities determines an algebra element $b_{0}(\phi) \in \mathbf{I}_{\mathbf{x}} \cdot \mathscr{B}_{0} \cdot \mathbf{I}_{\mathbf{y}}$, given by

$$
b_{0}(\phi)=U_{1}^{c_{1}(\phi)} \cdots U_{2 n}^{c_{2 n}(\phi)} \cdot \gamma_{\mathbf{x y}},
$$

where $c_{i}(\phi)=\min \left(n_{\ell_{i}}(\phi), n_{r_{i}}(\phi)\right)$.

Let $X$ denote the vector space spanned by upper Heegaard states. Consider the map

$$
\gamma_{0}^{1}: X \rightarrow \mathscr{B}_{0} \otimes X
$$

(again, where the tensor product is taken over the idempotent ring) defined by

$$
\gamma_{0}^{1}(\mathbf{x})=\sum_{\mathbf{y} \in \mathfrak{S}(\mathscr{H})} \sum_{\left\{\phi \in \pi_{2}(\mathbf{x}, \mathbf{y}) \mid \mu(\phi)=1\right\}} \#\left(\frac{\mathscr{M}(\phi)}{\mathbb{R}}\right) \cdot b_{0}(\phi) \cdot \mathbf{y} .
$$

Proposition 8.1. The endomorphism $\gamma_{0}^{1}$ satisfies the structure relation

$$
\left(\mu_{2}^{\mathscr{B}_{0}} \otimes \operatorname{Id}_{X}\right) \circ\left(\operatorname{Id}_{\mathscr{B}_{0}} \otimes \gamma_{0}^{1}\right) \circ \gamma_{0}^{1}(\mathbf{x})-\left(\sum_{\{i, j\} \in M} U_{i} U_{j}\right) \otimes \mathbf{x}+\mathscr{I} \otimes X,
$$

where $\mathscr{I}$ is the ideal used in the definition of $\mathscr{B}(2 n, n)$. 


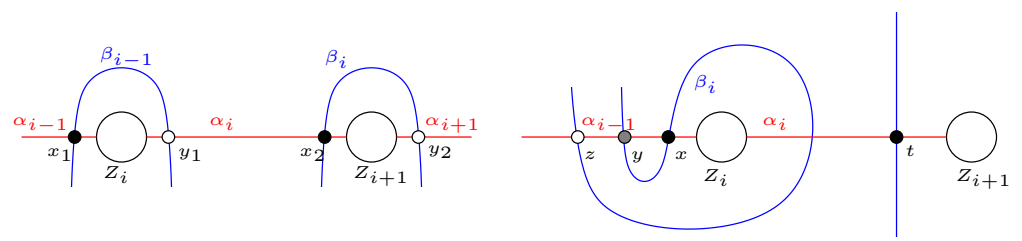

Figure 11. Relations in $\mathscr{B}$.

Sketch of proof. In broad terms, the proof of this is the usual $\partial^{2}=0$ proof in Lagrangian Floer homology: it is proved by considering one-dimensional moduli spaces of pseudo-holomorphic disks, and identifying their boundaries.

In more detail, the proof rests on the following observations

Observation 1. First, note that the map $b_{0}$ is additive under juxtapositions, in the sense that if $\mathbf{x}, \mathbf{y}, \mathbf{z} \in L_{\alpha} \cap \mathbb{T}_{\beta}, \phi_{1} \in \pi_{2}(\mathbf{x}, \mathbf{y})$, and $\phi_{2} \in \pi_{2}(\mathbf{y}, \mathbf{z})$ are two homotopy classes whose local multiplicities at all the $\ell_{i}$ and $r_{i}$ are non-negative, then

$$
b_{0}\left(\phi_{1} * \phi_{2}\right)=b_{0}\left(\phi_{1}\right) \cdot b_{0}\left(\phi_{2}\right) .
$$

This follows quickly from the fact that for any $\phi \in \pi_{2}(\mathbf{x}, \mathbf{y})$,

$$
n_{\ell_{i}}(\phi)-n_{r_{i}}(\phi)=v_{i}^{s(\mathbf{x})}-v_{i}^{s(\mathbf{y})},
$$

together with the additivity of local multiplicities under juxtapositions; i.e.

$$
n_{p}\left(\phi_{1} * \phi_{2}\right)=n_{p}\left(\phi_{1}\right)+n_{p}\left(\phi_{2}\right)
$$

for any $p \in \Sigma$, and the definition of multiplication in the algebra.

Observation 2. The next point is that if $\phi=\phi_{1} * \phi_{2}$, where $\phi_{1} \in \pi_{2}(\mathbf{x}, \mathbf{y})$ and $\phi_{2} \in \pi_{2}(\mathbf{y}, \mathbf{z})$ for some $\mathbf{y} \in L_{\alpha} \cap \mathbb{T}_{\beta}$ has an alternative decomposition $\phi=\phi_{1}^{\prime} * \phi_{2}^{\prime}$ with $\phi_{1} \in \pi_{2}\left(\mathbf{x}, \mathbf{y}^{\prime}\right)$ and $\phi_{2} \in \pi_{2}\left(\mathbf{y}^{\prime}, \mathbf{z}\right)$ with $\mathbf{y} \in\left(L_{\alpha}^{0} \cap \mathbb{T}_{\beta}\right) \backslash\left(L_{\alpha} \cap \mathbb{T}_{\beta}\right)$, then $b_{0}(\phi) \in \mathscr{I}$. To see why, we refer to Figure 11] At the left, the pair $\left\{x_{1}, x_{2}\right\}$ represents an upper state $\mathbf{x},\left\{x_{1}, y_{2}\right\}$ represents part of an upper state $\mathbf{y}$, and $\left\{y_{1}, y_{2}\right\}$ represents part of an upper state $\mathbf{z}$. The small bigon near $Z_{i+1}$ gives a term of $L_{i+1} \otimes \mathbf{y}$ in $\gamma_{0}^{1}(\mathbf{x})$; and the small bigon near $Z_{i}$ gives a term of $L_{i} \otimes \mathbf{z}$ in $\gamma_{0}^{1}(\mathbf{y})$. Since $L_{i+1} L_{i}=0$, we do not need to consider the ends of the moduli space from $\mathbf{x}$ to $\mathbf{z}$ : the corresponding term is in the ideal $\mathscr{I}$. Note that the alternative factorization of this moduli spaces involves $\left\{y_{1}, x_{2}\right\}$ which is not in a $L_{\alpha} \cap \boldsymbol{\beta}$. At the right is a similar picture, now with $\mathbf{x}$ containing $\{x, t\} \subset \mathbf{x},\{y, t\} \subset \mathbf{y}$, and $\{z, t\} \subset \mathbf{z}$. A small bigon from $x$ to $y$ gives a term of $\mathbf{y}$ in $\gamma_{0}^{1}(\mathbf{x})$. The bigon from $y$ to $z$ containing $Z_{i}$ gives a term of $U_{i} \otimes \mathbf{z}$ in $\gamma_{0}^{1}(\mathbf{y})$, which is in the ideal $\mathscr{I}$.

In view of the above two observations, it suffices to consider ends of moduli spaces $\phi \in \pi_{2}(\mathbf{x}, \mathbf{z})$ for which all broken flowline decompositions $\phi=\phi_{1} * \phi_{2}$ with $\phi_{1} \in$ $\pi_{2}(\mathbf{x}, \mathbf{y})$ and $\phi_{2} \in \pi_{2}(\mathbf{x}, \mathbf{y})$ have $\mathbf{y} \in L_{\alpha} \cap \mathbb{T}_{\beta}$. The usual $\partial^{2}=0$ proof now shows that the number of such ends has the same parity as the number of boundary degenerations: holomorphic curves which have boundary contained entirely on $L_{\alpha}^{0}$ or $\mathbb{T}_{\beta}$. We complete the proof with two more observations: 


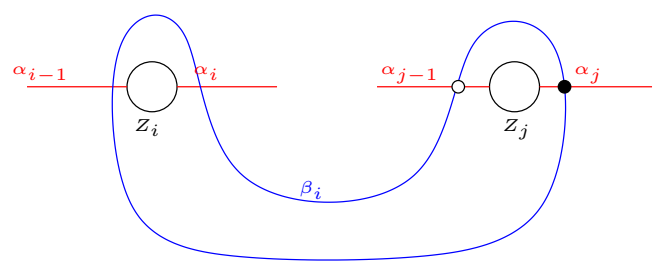

Figure 12. Motivation for introduction $C_{i, j}$.

Observation 3. A homotopy class corresponding to curves with boundary contained entirely in $\bar{L}_{\alpha}$ has positive coefficients at every $\ell_{i}$ and $m_{i}$; thus, the associated algebra element lies in the ideal $\mathscr{I}$.

And finally, to keep track of the $\beta$-boundary degenerations, we have:

Observation 4. There are $n$ homotopy classes of disks $\psi$ with boundary in $\mathbb{T}_{\beta}$, corresponding to the matchings $\{i, j\} \in M$, and their corresponding algebra element is $U_{i} \cdot U_{j}$; see Figure 12

The map $\gamma_{0}^{1}$ induces a map

$$
\gamma^{1}: X \rightarrow \mathscr{B} \otimes X
$$

satisfying the structure relation

$$
\left(\mu_{2} \otimes \operatorname{Id}_{X}\right) \circ\left(\operatorname{Id}_{\mathscr{B}} \otimes \gamma^{1}\right) \circ \gamma^{1}=\sum_{\{i, j\}} U_{i} U_{j}
$$

where here $\mu_{2}$ is multiplication in $\mathscr{B}$. Note that since $\mu_{1}=0$ in $\mathscr{B}$, the structure relation for $\gamma^{1}$ is nearly the type $D$ structure relation: it would be if the right hand side were zero. Instead, this structure can be thought of as a "curved" type $D$ structure (i.e. for an algebra with a $\mu_{0}$ operation); compare [49].

We can turn such an object into a type $D$ structure over $\mathcal{A}$, defining:

$$
\delta^{1}(\mathbf{x})=\left(\sum_{\{i, j\} \in M} C_{\{i, j\}}\right) \otimes \mathbf{x}+\gamma^{1}(\mathbf{x}) .
$$

This defines the type $D$ structure of an upper diagram.

We consider the upper diagram from Figure 9, Note that we are now working in the (first symmetric product of) the two-sphere. In some sense, the type $D$ structure is capturing the Lagrangian Floer homology of an interval with four marked points on it and the closed curve $\beta$. The type $D$ structure has five generators corresponding to the five intersection points, which we label from left to right in the picture 
$x_{1}, x_{2}, t, y_{1}, y_{2}$.

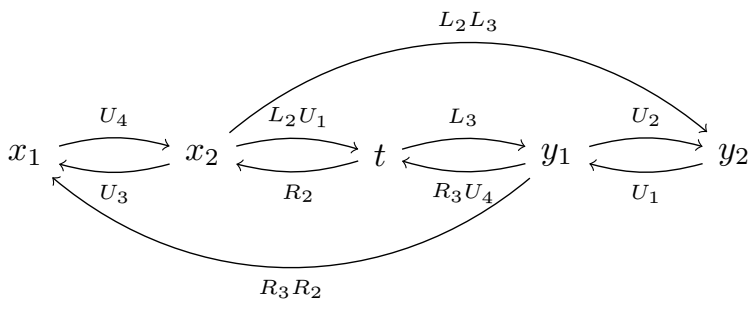

With a little more work, one can define the $\mathscr{A}_{\infty}$ module associated to a lower diagram. In this case, the higher actions count pseudo-holomorphic disks that go out to the $\alpha$-boundary. The algebra actions record the sequence of walls in which a pseudo-holomorphic disk crosses the walls in $L_{\alpha}$. Like in bordered Floer homology, it is clearer to express these actions in the language of Lipshitz's cylindrical reformulation of Heegaard Floer homology 62. A pairing theorem for recapturing knot Floer homology is then proved using a "time dilation" argument analogous to the bordered case (see [67, Chapter 9]), with a little extra attention paid now to $\beta$-boundary degenerations.

Working out the the type $D D$ bimodules for basic pieces is a fairly straightforward matter. Extending the pairing theorem to type $D A$ bimodules then gives the following:

Theorem 8.2. [85] If $K \subset S^{3}$ is a knot, then there are isomorphisms of bigraded modules

$$
\widehat{\operatorname{HFK}}(K) \cong \widehat{H}(K) \quad \operatorname{HFK}^{-}(K) \cong H^{-}(K) \quad \operatorname{HFK}(K) \cong H(K) .
$$

\section{FURTHER REMARKS}

To effectively compute the chain complexes $\mathscr{C}(K)$, one can start with the type $D$ structure corresponding to the global maximum, and successively enlarge it, moving down the knot projection. The computation is significantly improved by eliminating (by passing to a homotopy equivalent complex) generators $\mathbf{x}$ with

$$
\delta^{1}(\mathbf{x})=\sum_{\mathbf{y}} a_{\mathbf{x}, \mathbf{y}} \otimes \mathbf{y}
$$

for which some $a_{\mathbf{x}, \mathbf{y}}$ is an idempotent. Another simplification is achieved by working directly with the operators $\gamma^{1}$; see [86].

Throughout the above discussion, we worked in characteristic 2 to avoid signs. In fact, bordered knot Floer homology with $\mathbb{Z}$ coefficients can be worked out, after paying a little extra care to sign conventions. This is done in [86. One motivation is to find a knot $K$ in $S^{3}$ whose knot Floer homology (with $\mathbb{Z}$ coefficients) has torsion. Despite a rather extensive search, we have not yet found such a knot.

Long before the discovery of knot Floer homology, Andreas Floer proposed a construction of a knot invariant using instantons [31. In [58, Kronheimer and Mrowka conjectured an isomorphism between Floer's construction and knot Floer homology $\widehat{\mathrm{HFK}}$ (taken now with $\mathbb{Q}$ coefficients). This would provide some link between the fundamental group and knot Floer homology. 
The knot Floer homology package described here are sufficient for many computations: calculating $\tau(K), \epsilon(K)$, and $\widehat{\mathrm{HF}}$ of surgeries on $K$. To understand the function $\Upsilon(K)$ and $\mathrm{HF}^{-}$of surgeries on $K$, one needs to understand the knot Floer complex with more structure (in effect, without the $U V=0$ specialization from Equation (4.1)). To study this invariant, one must work with a larger algebra; see [87].

\section{REFERENCES}

[1] M. F. Atiyah. Floer homology, pages 105-108. Number 133 in Progr. Math. Birkhäuser, 1995.

[2] J. Baldwin and W. Gillam. Computations of Heegaard-Floer knot homology. J. Knot Theory Ramifications, 21(8):1250075, 65, 2012.

[3] D. Bar-Natan. On Khovanov's categorification of the Jones polynomial. Algebraic and Geometric Topology, 2:337-370, 2002.

[4] J. Batson. Nonorientable slice genus can be arbitrarily large. Math. Res. Lett., 21(3):423436, 2014.

[5] J. M. Bloom. A link surgery spectral sequence in monopole Floer homology. Adv. Math., 226(4):3216-3281, 2011.

[6] P. Braam and S. K. Donaldson. Floer's work on instanton homology, knots, and surgery. In H. Hofer, C. H. Taubes, A. Weinstein, and E. Zehnder, editors, The Floer Memorial Volume, number 133 in Progress in Mathematics, pages 195-256. Birkhäuser, 1995.

[7] W. Chen. On the upsilon invariant of cable knots. arXiv:1604.04760, 2016.

[8] D. H. Choi and K. H. Ko. Parametrizations of 1-bridge torus knots. J. Knot Theory Ramifications, 12(4):463-491, 2003.

[9] T. D. Cochran and R. E. Gompf. Applications of Donaldson's theorems to classical knot concordance, homology 3-spheres and property P. Topology, 27(4):495-512, 1988.

[10] V. Colin, P. Ghiggini, and K. Honda. Equivalence of Heegaard Floer homology and embedded contact homology via open book decompositions. Proc. Natl. Acad. Sci. USA, 108(20):8100-8105, 2011.

[11] J. H. Conway. An enumeration of knots and links, and some of their algebraic properties. In J. Leech, editor, Computational Problems in Abstract Algebra, pages 329-358. Pergamon, 1970.

[12] P. Cromwell. Embedding knots and links in an open book. I. Basic properties. Topology Appl., 64(1):37-58, 1995.

[13] H. Doll. A generalized bridge number for links in 3-manifolds. Math. Ann., 294(4):701-717, 1992.

[14] S. K. Donaldson. An application of gauge theory to four-dimensional topology. J. Differential Geom., 18(2):279-315, 1983.

[15] S. K. Donaldson. Irrationality and the $h$-cobordism conjecture. J. Differential Geom., 26(1):141-168, 1987.

[16] S. K. Donaldson. Polynomial invariants for smooth four-manifolds. Topology, 29(3):257-315, 1990.

[17] S. K. Donaldson. The Seiberg-Witten equations and 4-manifold topology. Bull. Amer. Math. Soc. (N.S.), 33(1):45-70, 1996.

[18] S. K. Donaldson. Lefschetz pencils on symplectic manifolds. J. Differential Geom., 53(2):205-236, 1999.

[19] S. K. Donaldson. Floer homology groups in Yang-Mills theory, volume 147 of Cambridge Tracts in Mathematics. Cambridge University Press, Cambridge, 2002. With the assistance of M. Furuta and D. Kotschick.

[20] S. K. Donaldson and P. B. Kronheimer. The Geometry of Four-Manifolds. Oxford Mathematical Monographs. Oxford University Press, 1990.

[21] S. Dostoglou and D. Salamon. Self-dual instantons and holomorphic curves. Ann. of Math., 2(139):581-640, 1994.

[22] I. Dynnikov. Arc-presentations of links: monotonic simplification. Fund. Math., 190:29-76, 2006. 
[23] Y. Eliashberg. Invariants in contact topology. In Proceedings of the International Congress of Mathematicians, Vol. II (Berlin, 1998), number Extra Vol. II, pages 327-338 (electronic), 1998.

[24] Y. Eliashberg and W. P. Thurston. Confoliations, volume 13 of University Lecture Series. AMS, Providence, RI, 1998.

[25] J. B. Etnyre. On symplectic fillings. Algebr. Geom. Topol., 4:73-80, 2004.

[26] P. Feller, J. Park, and A. Ray. On the upsilon invariant and satellite knots. arXiv/1604.04901.

[27] R. Fintushel and R. J. Stern. Definite 4-manifolds. J. Differential Geom., 28(1):133-141, 1988.

[28] R. Fintushel and R. J. Stern. Using Floer's exact triangle to compute Donaldson invariants, pages 435-444. Number 133 in Progr. Math. Birkhäuser, 1995.

[29] A. Floer. An instanton-invariant for 3-manifolds. Comm. Math. Phys., 119:215-240, 1988.

[30] A. Floer. Morse theory for Lagrangian intersections. J. Differential Geom., 28(3):513-547, 1988.

[31] A. Floer. Instanton homology, surgery, and knots. In Geometry of low-dimensional manifolds, 1 (Durham, 1989), volume 150 of London Math. Soc. Lecture Note Ser., pages 97-114. Cambridge Univ. Press, Cambridge, 1990.

[32] A. Floer. Instanton homology and Dehn surgery. In H. Hofer, C. H. Taubes, A. Weinstein, and E. Zehnder, editors, The Floer Memorial Volume, number 133 in Progress in Mathematics, pages 77-97. Birkhäuser, 1995.

[33] A. Floer, H. Hofer, and D. Salamon. Transversality in elliptic Morse theory for the symplectic action. Duke Math. J, 80(1):251-29, 1995.

[34] R. Friedman and J. W. Morgan. Smooth four-manifolds and complex surfaces, volume 27 of Ergebnisse der Mathematik und ihrer Grenzgebiete (3) [Results in Mathematics and Related Areas (3)]. Springer-Verlag, Berlin, 1994.

[35] K. A. Frøyshov. The Seiberg-Witten equations and four-manifolds with boundary. Math. Res. Lett, 3:373-390, 1996.

[36] K. Fukaya, Y-G. Oh, K. Ono, and H. Ohta. Lagrangian intersection Floer theory - anomaly and obstruction. Kyoto University, 2000.

[37] D. Gabai. Foliations and the topology of 3-manifolds. J. Differential Geom., 18(3):445-503, 1983.

[38] P. Ghiggini. Knot Floer homology detects genus-one fibred knots. Amer. J. Math., 130(5):1151-1169, 2008.

[39] H. Goda, H. Matsuda, and T. Morifuji. Knot Floer homology of $(1,1)$-knots. Geom. Dedicata, 112:197-214, 2005.

[40] R. E. Gompf and T. S. Mrowka. Irreducible 4-manifolds need not be complex. Ann. of Math. (2), 138(1):61-111, 1993.

[41] J. E. Greene. The lens space realization problem. Ann. of Math. (2), 177(2):449-511, 2013.

[42] M. Gromov. Pseudo holomorphic curves in symplectic manifolds. Inventiones mathematicae, 82:307-347, 1985.

[43] J. Hom. An infinite-rank summand of topologically slice knots. Geom. Topol., 19(2):1063$1110,2015$.

[44] A. Juhász. Holomorphic discs and sutured manifolds. Algebr. Geom. Topol., 6:1429-1457, 2006.

[45] A. Juhász. The sutured Floer homology polytope. Geom. Topol., 14(3):1303-1354, 2010.

[46] L. H. Kauffman. Formal knot theory. Number 30 in Mathematical Notes. Princeton University Press, 1983.

[47] B. Keller. A-infinity algebras, modules and functor categories. In Trends in representation theory of algebras and related topics, volume 406 of Contemp. Math., pages 67-93. Amer. Math. Soc., Providence, RI, 2006.

[48] M. Khovanov. A categorification of the Jones polynomial. Duke Math. J., 101(3):359-426, 2000.

[49] M. Khovanov and L. Rozansky. Matrix factorizations and link homology. Fund. Math., 199(1):1-91, 2008.

[50] M. Khovanov and P. Seidel. Quivers, Floer cohomology, and braid group actions. J. Amer. Math. Soc., 15(1):203-271, 2002. 
[51] M. Kontsevich. Homological algebra of mirror symmetry. In Proceedings of the International Congress of Mathematicians, Vol. 1, 2 (Zürich, 1994), pages 120-139. Birkhäuser, Basel, 1995.

[52] D. Kotschick. On manifolds homeomorphic to $\mathbf{C P}^{2} \# 8 \overline{\mathbf{C P}}^{2}$. Invent. Math., 95(3):591-600, 1989.

[53] P. Kronheimer and Tomasz Mrowka. Monopoles and three-manifolds, volume 10 of New Mathematical Monographs. Cambridge University Press, Cambridge, 2007.

[54] P. B. Kronheimer and T. Mrowka. Khovanov homology is an unknot-detector. Publ. Math. Inst. Hautes Études Sci., (113):97-208, 2011.

[55] P. B. Kronheimer and T. S. Mrowka. Gauge theory for embedded surfaces. I. Topology, 32(4):773-826, 1993.

[56] P. B. Kronheimer and T. S. Mrowka. Embedded surfaces and the structure of Donaldson's polynomial invariants. J. Differential Geom., 41(3):573-734, 1995.

[57] P. B. Kronheimer and T. S. Mrowka. Scalar curvature and the Thurston norm. Math. Res. Lett., 4(6):931-937, 1997.

[58] P. B. Kronheimer and T. S. Mrowka. Knots, sutures, and excision. J. Differential Geom., 84(2):301-364, 2010.

[59] P. B. Kronheimer, T. S. Mrowka, P. S. Ozsváth, and Z. Szabó. Monopoles and lens space surgeries. Ann. of Math. (2), 165(2):457-546, 2007.

[60] C. Kutluhan, Y-J. Lee, and C. H. Taubes. $H F=H M$ I: Heegaard Floer homology and Seiberg-Witten Floer homology. 1007.1979, 2010.

[61] E. S. Lee. An endomorphism of the Khovanov invariant. Adv. Math., 197(2):554-586, 2005.

[62] R. Lipshitz. A cylindrical reformulation of Heegaard Floer homology. Geom. Topol., 10:9551097 (electronic), 2006.

[63] R. Lipshitz, P. S. Ozsváth, and D. P. Thurston. Heegaard Floer homology as morphism spaces. Quantum Topol., 2(4):381-449, 2011.

[64] R. Lipshitz, P. S. Ozsváth, and D. P. Thurston. Tour of bordered Floer theory. Proc. Natl. Acad. Sci. USA, 108(20):8085-8092, 2011.

[65] R. Lipshitz, P. S. Ozsváth, and D. P. Thurston. Computing $\widehat{H F}$ by factoring mapping classes. Geom. Topol., 18(5):2547-2681, 2014.

[66] R. Lipshitz, P. S. Ozsváth, and D. P. Thurston. Bimodules in bordered Heegaard Floer homology. Geom. Topol., 19(2):525-724, 2015.

[67] R. Lipshitz, D. P. Thurston, and P. S. Ozsváth. Bordered Floer homology. arXiv:0810.0687.

[68] R. Litherland. Signatures of iterated torus knots. In Topology of low-dimensional manifolds (Proc. Second Sussex Conf., Chelwood Gate, 1977), volume 722 of Lecture Notes in Math., pages 71-84. Springer, Berlin, 1979.

[69] C. Livingston. Computations of the Ozsváth-Szabó knot concordance invariant. Geom. Topol., 8:735-742 (electronic), 2004.

[70] C. Livingston. Notes on the knot concordance invariant upsilon. Algebr. Geom. Topol., 17(1):111-130, 2017.

[71] A. Manion. Khovanov-Seidel quiver algebras and Ozsváth-Szabó's bordered theory. Journal of Algebra, 2017. arXiv:1605.08082.

[72] C. Manolescu. Seiberg-Witten-Floer stable homotopy type of three-manifolds with $b_{1}=0$. Geom. Topol., 7:889-932 (electronic), 2003.

[73] C. Manolescu and P. S. Ozsváth. On the Khovanov and knot Floer homologies of quasialternating links. In Proceedings of Gökova Geometry-Topology Conference 2007, pages 60-81. Gökova Geometry/Topology Conference (GGT), Gökova, 2008.

[74] C. Manolescu, P. S. Ozsváth, and S. Sarkar. A combinatorial description of knot Floer homology. Ann. of Math. (2), 169(2):633-660, 2009.

[75] C. Manolescu, P. S. Ozsváth, Z. Szabó, and D. Thurston. On combinatorial link Floer homology. Geom. Topol., 11:2339-2412, 2007.

[76] M. Marcolli and B-L. Wang. Equivariant Seiberg-Witten Floer homology. Comm. Anal. Geom., 9(3):451-639, 2001.

[77] J. Milnor. Morse theory. Based on lecture notes by M. Spivak and R. Wells. Annals of Mathematics Studies, No. 51. Princeton University Press, Princeton, N.J., 1963.

[78] J. Milnor. Lectures on the $h$-cobordism theorem. Princeton University Press, 1965. Notes by L. Siebenmann and J. Sondow. 
[79] J. Milnor. Singular points of complex hypersurfaces. Annals of Mathematics Studies, No. 61. Princeton University Press, Princeton, N.J., 1968.

[80] K. Murasugi. On the Alexander polynomial of alternating algebraic knots. J. Austral. Math. Soc. Ser. A, 39(3):317-333, 1985.

[81] L. Ng, P. S. Ozsváth, and D. Thurston. Transverse knots distinguished by knot Floer homology. J. Symplectic Geom., 6(4):461-490, 2008.

[82] Y. Ni. Knot Floer homology detects fibred knots. Invent. Math., 170(3):577-608, 2007.

[83] P. S. Ozsváth, A. Stipsicz, and Z. Szabó. Unoriented knot Floer homology and the unoriented four-ball genus. arXiv:1508.03243, 2015.

[84] P. S. Ozsváth, A. I. Stipsicz, and Z. Szabó. Grid homology for knots and links, volume 208 of Mathematical Surveys and Monographs. American Mathematical Society, Providence, RI, 2015.

[85] P. S. Ozsváth and Z. Szabó. Algebras with matchings and knot Floer homology. In preparation.

[86] P. S. Ozsváth and Z. Szabó. Bordered knot algebras with matchings. Preprint.

[87] P. S. Ozsváth and Z. Szabó. The pong algebra. In preparation.

[88] P. S. Ozsváth and Z. Szabó. Heegaard Floer homology and alternating knots. Geom. Topol., 7:225-254, 2003.

[89] P. S. Ozsváth and Z. Szabó. Knot Floer homology and the four-ball genus. Geom. Topol., 7:615-639, 2003.

[90] P. S. Ozsváth and Z. Szabó. Holomorphic disks and genus bounds. Geom. Topol., 8:311-334, 2004.

[91] P. S. Ozsváth and Z. Szabó. Holomorphic disks and knot invariants. Adv. Math., 186(1):58116, 2004.

[92] P. S. Ozsváth and Z. Szabó. Holomorphic disks and topological invariants for closed threemanifolds. Ann. of Math. (2), 159(3):1027-1158, 2004.

[93] P. S. Ozsváth and Z. Szabó. Holomorphic triangle invariants and the topology of symplectic four-manifolds. Duke Math. J., 121(1):1-34, 2004.

[94] P. S. Ozsváth and Z. Szabó. On knot Floer homology and lens space surgeries. Topology, 44(6):1281-1300, 2005.

[95] P. S. Ozsváth and Z. Szabó. Holomorphic disks, link invariants and the multi-variable Alexander polynomial. Algebr. Geom. Topol., 8(2):615-692, 2008.

[96] P. S. Ozsváth and Z. Szabó. Knot Floer homology and integer surgeries. Algebr. Geom. Topol., 8(1):101-153, 2008.

[97] P. S. Ozsváth and Z. Szabó. Knot Floer homology and rational surgeries. Algebr. Geom. Topol., 11(1):1-68, 2011.

[98] P. S. Ozsváth and Z. Szabó. Kauffman states, bordered algebras, and a bigraded knot invariant. arXiv:1603.06559, 2016.

[99] P. S. Ozsváth, Z. Szabó, and A. Stipsicz. Concordance homomorphisms from knot Floer homology. arXiv:1407.1795, 2014.

[100] P. S. Ozsváth, Z. Szabó, and D. Thurston. Legendrian knots, transverse knots and combinatorial Floer homology. Geom. Topol., 12(2):941-980, 2008.

[101] T. Perutz. Hamiltonian handleslides for Heegaard Floer homology. In Proceedings of Gökova Geometry-Topology Conference 2007, pages 15-35. Gökova Geometry/Topology Conference (GGT), Gökova, 2008.

[102] I. Petkova and V. Vértesi. Combinatorial tangle Floer homology. Geom. Topol., 20(6):32193332,2016

[103] A. Polishchuk and L. Positselski. Quadratic algebras, volume 37 of University Lecture Series. American Mathematical Society, Providence, RI, 2005.

[104] J. A. Rasmussen. Floer homology of surgeries on two-bridge knots. Algebr. Geom. Topol., 2:757-789 (electronic), 2002.

[105] J. A. Rasmussen. Floer homology and knot complements. PhD thesis, Harvard University, 2003.

[106] J. A. Rasmussen. Khovanov homology and the slice genus. Invent. Math., 182(2):419-447, 2010.

[107] J. Robbin and D. Salamon. The spectral flow and the Maslov index. Bull. London Math. Soc., 27(1):1-33, 1995. 
[108] S. Sarkar. Grid diagrams and the Ozsváth-Szabó tau-invariant. Math. Res. Lett., 18(6):1239$1257,2011$.

[109] S. Sarkar and J. Wang. An algorithm for computing some Heegaard Floer homologies. Ann. of Math. (2), 171(2):1213-1236, 2010.

[110] P. Seidel. Fukaya categories and Picard-Lefschetz theory. Zurich Lectures in Advanced Mathematics. European Mathematical Society (EMS), Zürich, 2008.

[111] J. D. Stasheff. Homotopy associativity of $H$-spaces. I. Trans. Amer. Math. Soc. 108 (1963), 275-292, 108:275-292, 1963.

[112] Z. Szabó. Simply-connected irreducible 4-manifolds with no symplectic structures. Invent. Math., 132(3):457-466, 1998.

[113] C. H. Taubes. Casson's invariant and gauge theory. J. Differential Geom., 31(2):547-599, 1990.

[114] C. H. Taubes. The Seiberg-Witten invariants and symplectic forms. Math. Res. Lett., 1(6):809-822, 1994

[115] C. H. Taubes. More constraints on symplectic forms from Seiberg-Witten invariants. Math. Res. Lett., 2(1):9-13, 1995.

[116] C. H. Taubes. SW $\rightarrow$ Gr: from the Seiberg-Witten equations to pseudo-holomorphic curves. J. Amer. Math. Soc., 9(3):845-918, 1996.

[117] K. Wehrheim. Lagrangian boundary conditions for anti-self-dual instantons and the AtiyahFloer conjecture. J. Symplectic Geom., 3(4):703-747, 2005. Conference on Symplectic Topology.

[118] E. Witten. Monopoles and four-manifolds. Math. Res. Lett., 1(6):769-796, 1994.

[119] R. Zarev. Bordered Sutured Floer Homology. ProQuest LLC, Ann Arbor, MI, 2011. Thesis (Ph.D.)-Columbia University

[120] B. Zhan. Combinatorial proofs in bordered Heegaard Floer homology. Algebr. Geom. Topol., 16(5):2571-2636, 2016.

Department of Mathematics, Princeton University, Princeton, New Jersey 08544

E-mail address: petero@math.princeton.edu

Department of Mathematics, Princeton University, Princeton, New Jersey 08544

E-mail address: szabo@math.princeton.edu 